\title{
Quasi-Euclidean classification of Alcoved Convex Polyhedra*
}

\author{
M.J. de la Puente ${ }^{\dagger}$ \\ Dpto. de Álgebra, Geometría y Topología \\ Facultad de Matemáticas \\ Universidad Complutense \\ mpuenteducm.es \\ Phone: 34-91-3944659
}

\begin{abstract}
We give the quasi-Euclidean classification of the maximal (with respect to the $f$-vector) alcoved polyhedra. The $f$-vector of these maximal convex bodies is $(20,30,12)$, so they are simple dodecahedra. We find eight quasiEuclidean classes. This classification, which preserves angles, is finer than the known combinatorial classification (found in 2012 by Jiménez and de la Puente), which has only six classes. Each alcoved polyhedron $\mathcal{P}$ is represented by a unique visualized idempotent matrix $A$. Some 2-minors of $A$ are invariants of $\mathcal{P}$ : they are the tropical edge-lengths of $\mathcal{P}$.

Keywords: tropical algebra; max-plus algebra; normal matrix; idempotent matrix; visualized matrix; alcoved polyhedron; dodecahedron; convex body; quasi-Euclidean class; perturbation; invariant, edge-length; tropical distance.
\end{abstract}

AMS Classification: 52B10; 52B12; $15 \mathrm{~A} 80$

\section{Introduction}

Alcoved polyhedra in $\mathbb{R}^{3}$ make a rich, very well-behaved and beautiful class of convex bodies. They have facet equations only of two types: $x_{i}=$ cnst and $x_{i}-$ $x_{j}=$ cnst. Two consequences follow. First, the $f$-vector, the facet sequence, the facet angles and the dihedral angles are restricted. Second, the constant terms of the facet equations can be assembled into a matrix, leading to an algebraization of alcoved polyhedra.

*DOI: $10.1080 / 03081087.2019 .1572065$

†Partially supported by Ministerio de Economía y Competitividad, Proyecto I+D MTM201676808-P and by UCM research group 910444. 
Every alcoved polyhedron $\mathcal{P} \subset \mathbb{R}^{3}$ can be represented by a normal idempotent (NI) order four matrix $A$. We write $\mathcal{P}=\mathcal{P}(A)$ to indicate this relationship. To a certain translate of $\mathcal{P}$ there corresponds a unique matrix, which in addition to the former properties, is visualized (VI). In this paper we show that an alcoved polyhedron $\mathcal{P}$ can be handled by handling its corresponding matrix $A$.

The $f$-vector of a polyhedron $\mathcal{P}$ lists the number of vertices, edges and facets of $\mathcal{P}$. For an alcoved one, it is known to be bounded above by $(20,30,12)$. Maximal alcoved polyhedra are those having such maximal $f$-vector. They are simple (i.e., trivalent in vertices) dodecahedra with 20 vertices and 30 edges.

In this paper we classify alcoved polyhedra from a topological, affine and Euclidean point of view, i.e., we transform them by small perturbations, affine maps and space symmetries. Here is what we do. First, we declare all boxes to be equivalent (disregarding length). Having done so, we are left with the classification of alcoved polyhedra having a common bounding box. Say this common bounding box is $\mathcal{Q}$, the unit cube centered at the origin. Two alcoved polyhedra $\mathcal{P}$ and $\mathcal{P}^{\prime}$ whose bounding box is $\mathcal{Q}$ are equivalent for us, in two cases: if there exists a symmetry of $\mathcal{Q}$ taking $\mathcal{P}$ bijectively onto $\mathcal{P}^{\prime}$ or if $\mathcal{P}^{\prime}$ is a sufficiently small perturbation of $\mathcal{P}$ (or a combination of both). Finally, when we want to compare two arbitrary alcoved polyhedra $\mathcal{P}$ and $\mathcal{P}^{\prime}$, first we transform their bounding boxes $\mathcal{B}$ and $\mathcal{B}^{\prime}$ into $\mathcal{Q}$ by bijective affine maps $f$ and $f^{\prime}$. If $f(\mathcal{P})$ and $f^{\prime}\left(\mathcal{P}^{\prime}\right)$ are equivalent,as described above, then we define the original $\mathcal{P}$ and $\mathcal{P}^{\prime}$ to be equivalent. It is an angle-preserving equivalence relation between maximal alcoved polyhedra. We have composed the expression quasi-Euclidean equivalence to refer to such a relation.

Trivially, in $\mathbb{R}^{2}$ the maximal alcoved polygons are hexagons (slopes of sides being $0,1,+\infty$ ) and there exists just one quasi-Euclidean class of such. In $\mathbb{R}^{3}$ the maximal alcoved polyhedra are dodecahedra. Our main theorem goes as follows

Theorem 1.1. The quasi-Euclidean classification in the family of maximal alcoved dodecahedra in $\mathbb{R}^{3}$ has eight classes.

Every non-maximal alcoved polyhedron arises from a maximal one, when some edges collapse, and simplicity is lost. Instances of such are boxes. With similar arguments, the quasi-Euclidean classification of non-maximal alcoved polyhedra is possible to devise, but we believe it is far less interesting (and far more involved).

The proof of the main theorem is completed in p. 32. It is reached after the following steps. In each alcoved polyhedron $\mathcal{P}$, two distinguished vertices are called North and South Poles, and marked $\mathcal{N}$ and $\mathcal{S}$. After an idea of Kepler's, the polyhedron $\mathcal{P}$ is the union of three parts: North Cask, South Cask and Equatorial Belt. For instance, each box has a trivial Equatorial Belt. Each Polar Cask has a Cask type. Cask types are described by a vector and a chirality word. In the maximal case, the vector is (p.q.r), with $p, q, r \in\{4,5,6\}$ and $p+q+r=15$. The 
chirality word is either left or right and it is omitted whenever it can be deduced. In the maximal case, we show that both the Equatorial Belt and the quasi-Euclidean class are determined only by the North and South Cask types. The Cask types are determined only by the signs of six 2 -minors of the NI matrix $A$ representing $\mathcal{P}$. Ultimately, the Cask types are determined by the signs of a 6-tuple, called difference tuple, which is computed from the perturbation matrix $E$ of $A$ in a very direct way (see example 9.18).

The classes are denoted $Q E 1, Q E 2, \ldots, Q E 8$ and the classification is shown in table 1, which reads as follows. The rectangle on the first row left describes a representative of class $Q E 1$ : it is a dodecahedron having a North Cask type vector (4.5.6) and a South Cask type vector (4.5.6). This means that the North Cask is composed of the facet $x_{1}=$ cnst, which is a 4-gon, the facet $x_{2}=$ cnst a 5-gon, and the facet $x_{3}=$ cnst a 6 -gon and they all meet at $\mathcal{N}$. The chirality word here can be deduced from the former data and has been omitted. It is left. In the South Cask there is a facet $x_{1}=$ cnst, which is a 4-gon, a facet $x_{2}=$ cnst a 5-gon, and a facet $x_{3}=$ cnst a 6 -gon. Similarly for other rectangles in the table.

\begin{tabular}{|l|l|l|l|l|}
\hline $\mathcal{N}(4.5 .6)$ & $\mathcal{N}(4.5 .6)$ & $\mathcal{N}(4.5 .6)$ & $\mathcal{N}(4.5 .6)$ & $\mathcal{N}(4.5 .6)$ \\
$\mathcal{S}(4.5 .6)$ & $\mathcal{S}(5.6 .4)$ & $\mathcal{S}(6.5 .4)$ & $\mathcal{S}(4.6 .5)$ & $\mathcal{S}(5.4 .6)$ \\
$Q E 1$ & $Q E 2$ & $Q E 3$ & $Q E 4$ & $Q E 5$ \\
\hline & $\mathcal{N}(5.5 .5)$ right & $\mathcal{N}(4.5 .6)$ left & $\mathcal{N}(5.4 .6)$ right & \\
& $\mathcal{S}(5.5 .5)$ left & $\mathcal{S}(5.5 .5)$ left & $\mathcal{S}(5.5 .5)$ left & \\
& $Q E 6$ & $Q E 7$ & $Q E 8$ & \\
\hline
\end{tabular}

Table 1: The eight quasi-Euclidean classes of maximal alcoved dodecahedra in $\mathbb{R}^{3}$. In each case, the third line shows the class name. The first and second lines describe the North and South Cask types of one representative of the class.

Alcoved polyhedra may have pentagonal facets and, as soon as pentagons appear, chirality appears too. Chirality changes the orientation. A chiral copy of $\mathcal{P}(A)$ belongs to the same quasi-Euclidean class. Algebraically, we produce a chiral copy of $\mathcal{P}(A)$ by letting some group element act on the matrix $A$.

Our starting point is the known fact that a normal idempotent of order $n$ matrix $A$ provides an alcoved polytope $\mathcal{P}(A)$ in $\mathbb{R}^{n-1}$, idempotency with respect to tropical multiplication. Translations of $\mathcal{P}(A)$ correspond to conjugations of $A$ by diagonal matrices. It is particularly nice when $\mathcal{P}$ is cornered, meaning that the largest point in $\mathcal{P}=\mathcal{P}(A)$ is the origin. In such a case, the corresponding matrix $A$ is visualized idempotent $(\mathrm{VI})$ matrix, a very nice matrix to compute with.

Consider $n=4$. For a VI matrix $A$, we prove that the tropical edge-lengths of $\mathcal{P}(A)$ are equal to either the entries of the box matrix $B$ or the entries of the 
perturbation matrix $E$, where $A$ is decomposed as the sum $A=B-E$ (classical sum here). This algebraization of the edge-lengths of $\mathcal{P}(A)$ is a key fact. We prove that all these entries (of $B$ and of $E$ ) are 2-minors of $A=B-E$. The 2-minors are certain sums and differences of matrix entries.

We prove that 2 -minors are preserved by visualization. This means that the edge-lengths of $\mathcal{P}(A)$ are certain 2-minors of $A$, when $A$ is only NI (but perhaps not VI, and $A$ does not admit a box-perturbation decomposition). In other words, certain 2-minors of $A$ are metric invariants of $\mathcal{P}(A)$, for any NI order 4 matrix $A$.

For $A=B-E$, the bounding box of $\mathcal{P}(A)$ is just $\mathcal{P}(B)$ and the cant and difference tuples $c$ and $d \in \mathbb{R}^{6}$ are obtained easily from the matrix $E$. Both $B$ and $c$ carry the metric information of the polyhedron $\mathcal{P}(A)$. The difference tuple $d$ carries the quasi-Euclidean class information of $\mathcal{P}(A)$. With these tools, we first study the North Cask of $\mathcal{P}(A)$. Then, we study the South Cask of $\mathcal{P}(A)$, by turning $\mathcal{P}(A)$ around by a Polar Exchange, which amounts to transforming the matrix $A$ under the action of some group element.

Our classification is both geometrical (in the sense of Euclidean geometry) and topological. Indeed, for polyhedra whose bounding box is the unit cube $\mathcal{Q}$, we say that two alcoved polyhedron $\mathcal{P}, \mathcal{P}^{\prime}$ are equivalent whenever either a cube symmetry takes $\mathcal{P}$ onto $\mathcal{P}^{\prime}$ or when $\mathcal{P}^{\prime}$ is a small perturbation of $\mathcal{P}$, or both. The group of cube symmetries relevant here, denoted $\mathcal{G}_{3}$, is isomorphic to $\mathbb{Z}_{2} \times \Sigma_{3}$. Here $\Sigma_{3}$ denotes the permutation group in 3 symbols.

The ideas of this paper have been applied to exact volume computation in [41]. Our techniques might admit a generalization to alcoved polytopes in higher dimensions, but it is certainly not straightforward. Thus we have a sound reason to work today with alcoved polyhedra in 3-dimensional space. Other more general alcoved polytopes arise from root systems. There is a connection between alcoved polyhedra and the root system $A_{3}$. Indeed, the facet exterior normal vectors of an alcoved polyhedron are $\left\{ \pm u_{i}: i \in[3]\right\} \cup\left\{ \pm\left(u_{i}-u_{j}\right): i \neq j \in[3]\right\}$, a proper subset of the $A_{3}$ roots, which are $\left\{ \pm u_{i}: i \in[3]\right\} \cup\left\{ \pm u_{i} \pm u_{j}: i \neq j \in[3]\right\}$. Here $\left(u_{1}, u_{2}, u_{3}\right)$ denotes the canonical basis in $\mathbb{R}^{3}$.

Alcoved polytopes have been studied in great generality since 2007, from a combinatorial point of view; see [30, 31, 42]. In relation with Tropical Mathematics, they appeared earlier in connection with max-plus spectral theory and matrix eigenspaces in [2, 3, 4] (see also the bibliography in the survey [2]). In relation to classical convex polytopes and polytopal complexes, they have been studied in the foundational paper [20], and subsequent papers [26, 27, 28, 39, 53, 54]. In the recent book [9], it is shown that alcoved polytopes have a crystal base (or Kashiwara crystal) structure. In relation with Jacobian curves, alcoved polytopes have been used in [57], in order to prove a classical result on algebraic curves by means of tropical techniques.

Normal matrices (and slightly weaker notions) have been studied for more 
than fifty years (see the pioneer works [1, 8, 55]), under various different names. In connection with Tropical Mathematics, they appear in [12, 13, 14, 15, 39, 48] and very recently in [56]. In connection with Automata Theory, Scheduling Theory, Computer Science, Discrete Event Systems and Dynamical Programming, normal matrices appear in [6, 7, 22, 36] among other, where they are named difference bounded matrices (DBM). Visualization of matrices is one way to standardize normal idempotent matrices, appearing in [2, 49]. Tropical Mathematics is an emerging area with multiple connections to Analysis, Algebraic Geometry, Representations of Lie Algebras, Mathematical Economics and Algebra: see $[10,11,24,25,32,33,34,35,44,52]$ and bibliographies therein.

The paper is organized as follows. In section 2 we introduce left and right pentagons, North and South Poles, and some combinatorial labels of vertices of an alcoved polyhedron. In section 3 we present boxes and cubes, together with their matrices. These are the easiest alcoved polyhedra and the easiest matrices. More general matrices are introduced in section 4. In this section we gather the core facts: we restrict ourselves to working with normal idempotent matrices (NI) because they provide alcoved polytopes (see the pioneer paper [20] on tropical convexity vs. classical convexity). Even more, if we restrict ourselves to visualized idempotent matrices $A$ (VI for short), then the edge-lengths of the corresponding alcoved polyhedron (which is cornered) can easily be read from the decomposition $A=B-E$ of the matrix. In a subsection, we introduce the useful notion of 2-minors of a matrix $A$. Some of them are edge-lengths of $\mathcal{P}(A)$. In section 5 we introduce bounding boxes, perturbations and cant and difference tuples. More precisely, every alcoved polytope is the perturbation of a box, and every box is an unperturbed alcoved polyhedron. The perturbation consists in canting (i.e., beveling) a cycle of box edges. In section 6 we introduce the subgroup, denoted $\mathcal{G}_{3}$, of cube symmetries fixing a given diagonal. This group acts on the set $M_{4}^{N I}$ of normal idempotent matrices of order four. In the short sections 7 and 8 we make precise the notions of maximality and of tropical distance. Section 9 contains the heavy work: we define North and South Casks and we show that Cask types of a maximal polyhedron $\mathcal{P}(A)$ depend on the signs of certain 2-minors of $A$. Ultimately, the Cask types depend on the signs of the difference tuple $d$ of $A$. The quasi-Euclidean classification is presented in section 10. There is an action of a group isomorphic to $\mathcal{G}_{3}$ on a set of sign 6tuples. The orbit of $\operatorname{sign}(d)$, the sign of the difference tuple $d$ of $A$, provides the equivalence class of $\mathcal{P}(A)$. A worked example is given in p. 29 .

The relationship of the present classification with the coarser combinatorial classification (found in 2012 by Jiménez and de la Puente [26]) is shown in table 7. Positive answers (in the realm of alcoved polyhedra) to two open questions are given in section ??.

The number 8 plays two roles in this paper: on the one hand, it is the number of North and South Cask Types and, on the other hand, it is the number of quasi- 
Euclidean clases.

Notations and colors: in figures, the origin is marked with a red dot and generators are marked with blue dots, three-generated vertices are marked with magenta dots, two-generated vertices are marked with yellow dots. The unit cube centered at the origin is denoted $\mathcal{Q} \subset \mathbb{R}^{3}$. Vectors and points will be written in columns. Given $n \in \mathbb{N}$, the set $\{1,2, \ldots, n\}$ is denoted $[n]$. Let $\Sigma_{n}$ denote the permutation groups in $n$ symbols.
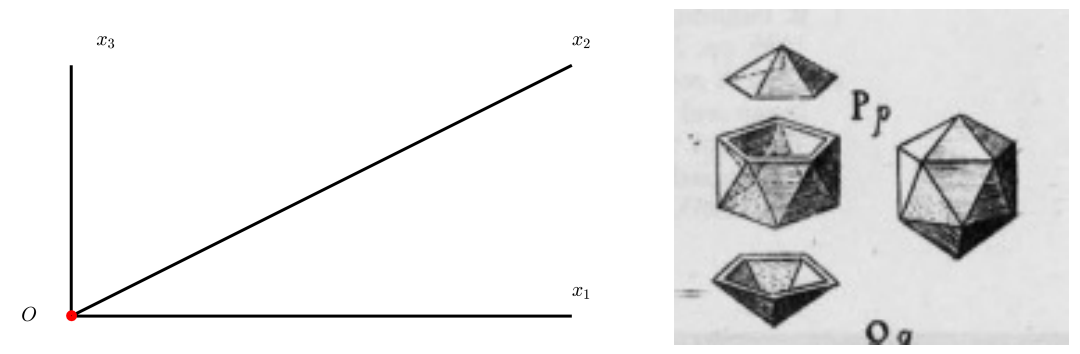

Figure 1: Coordinate axes in $\mathbb{R}^{3}$; the red dot marks the origin (left). North and South Casks and Equatorial Belt in an icosahedron in figure from Kepler's Harmonices Mundi, 1691 (right).

\section{Left and right pentagons, geographical terms and some combinatorial labels}

Definition 2.1 (Left pentagon and right pentagon). Consider a rectangle $F \subset \mathbb{R}^{2}$ with edges parallel to the coordinate axes and fix a vertex $\mathcal{V}$ of $F$ such that $\mathcal{V}=$ $\max R$ or $\mathcal{V}=\min R$, coordinatewise. Cut off a corner of $F$ not opposite to $\mathcal{V}$ and obtain a pentagon $F^{\prime}$. We say that $F^{\prime}$ is a left pentagon if the removed corner is on the left hand side of $F$. Otherwise we say $F^{\prime}$ is a right pentagon.

Two distinguished vertices exist in every alcoved polyhedron $\mathcal{P} \subset \mathbb{R}^{3}$ : the North and South Poles. These are $\mathcal{N}=\max \mathcal{P}$ and $\mathcal{S}=\min \mathcal{P}$, where maxima and minima are computed coordinatewise. The classical line passing through $\mathcal{N}$ and $\mathcal{S}$ will be called the Polar Axis of $\mathcal{P}$. The largest point in $\mathcal{P}$ is the North Pole. The smallest point in $\mathcal{P}$ is the South Pole.

In addition, we use a combinatorial labeling of vertices, marked with underlined digits. The single-digit vertices of $\mathcal{P}$ are called generators (marked blue in figures); there are four of them: $j$, for $j \in[4]$.

The labels of the remaining vertices of $\mathcal{P}$ may have 2 or 3 digits. A vertex in $\mathcal{P}$ may admit two or more labels and this happens if and only if $\mathcal{P}$ is non-maximal (with respect to $f$-vector). More combinatorial labels are introduced in p. 12 . 


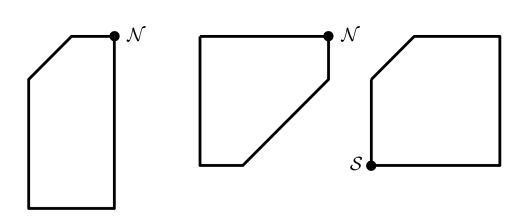

Figure 2: A left pentagon at the North Pole is such that the left corner of a rectangle is missing (left). A right pentagon at the North Pole: the right corner has been cut off (center). A left pentagon at the South Pole: the left corner has been cut off (right).

\section{Boxes and Cubes}

We identify $\mathbb{R}^{n-1}$ with the hyperplane $\left\{x_{n}=0\right\}$ in $\mathbb{R}^{n}$.

Definition 3.1. A box in $\mathbb{R}^{n-1}$ is a polytope $\mathcal{B}$ whose facets are contained in hyperplanes parallel to the coordinate axes. A cube is a box of equal edge-lengths.

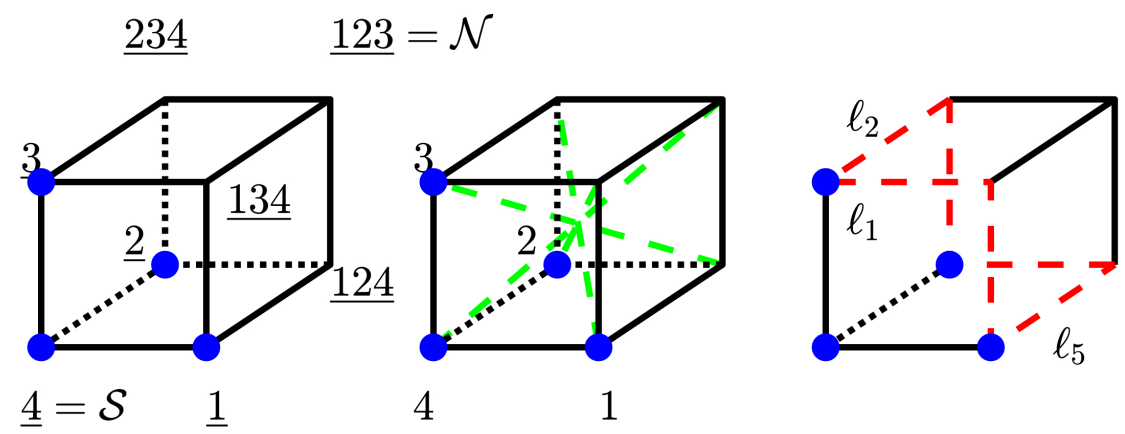

Figure 3: In $\mathbb{R}^{3}$, labeled vertices of a cube, using underlined single-digit or underlined three-digit labels (left). Labeled diagonals of a cube (diagonals marked in green dashed lines) (center). Cantable edges $\ell_{1}, \ldots, \ell_{6}$ in a cube (right). They form a cycle, beginning with $\ell_{1}$ in front top, $\ell_{2}$ top left, $\ell_{3}$ left back, $\ell_{4}$ back bottom, $\ell_{5}$ bottom right and $\ell_{6}$ right front. Cantable edges marked in red dashed lines.

Definition 3.2 (Labels in a box). 1. The vertices of a 3-dimensional box are labeled $j$, with $j \in[4]$ and $123, \underline{124}, \underline{134}, \underline{234}$. In particular, 123 is the North Pole and $\underline{4}$ is the South Pole.

2. We label the diagonals of boxes with plain (not underlined) digits as follows: the diagonal $j$ contains the generator $j$, for $j \in[4]$ (see figure 3 , center). In particular, the label of the Polar Axis is 4. 
For $j \in[3]$ consider the facet $F_{j}$ of a box all whose vertices contain $j$ in the label. Then, it is easy to see that $\underline{j}$ is the smallest point of $F_{j}$.

Notation 3.3. Given a vector $t=\left(t_{1}, t_{2}, t_{3}\right)^{T} \in \mathbb{R}_{\leq 0}^{3}$, consider the following matrix

$$
B(t):=\left(\begin{array}{rrrr}
0 & t_{1} & t_{1} & t_{1} \\
t_{2} & 0 & t_{2} & t_{2} \\
t_{3} & t_{3} & 0 & t_{3}
\end{array}\right) .
$$

Since $\mathbb{R}^{3}$ is identified with $\left\{x_{4}=0\right\}$ in $\mathbb{R}^{4}$, then the matrix $B(t)$ is identified with the matrix

$$
\left(\begin{array}{rrrr}
0 & t_{1} & t_{1} & t_{1} \\
t_{2} & 0 & t_{2} & t_{2} \\
t_{3} & t_{3} & 0 & t_{3} \\
0 & 0 & 0 & 0
\end{array}\right)
$$

It is easy to check that the columns of $B(t)$ are the generators of a box $\mathcal{B} \subset \mathbb{R}^{3}$ whose North Pole is the origin and whose edge-lengths are $\left|t_{1}\right|,\left|t_{2}\right|,\left|t_{3}\right|$. We say that the matrix $B(t)$ represents the box $\mathcal{B}$.

\section{Alcoved convex polyhedra and their matrices}

In the previous section we have seen that certain matrices represent boxes. Here we will see matrices which represent alcoved polyhedra. Let $x_{1}, \ldots, x_{n-1}$ be coordinates in $\mathbb{R}^{n-1}$.

Definition 4.1 (Alcoved). A convex polytope $\mathcal{P}$ in $\mathbb{R}^{n-1}$ is alcoved if the facets of $\mathcal{P}$ are contained in hyperplanes of equations $x_{i}=$ cnst or $x_{i}-x_{j}=$ cnst, for $i, j \in[n-1]$.

In particular, a box is a non-maximal (with respect to $f$-vector) alcoved polytope.

Definition 4.2 (Cornered alcoved polytope). An alcoved polytope $\mathcal{P} \subset \mathbb{R}^{n-1}$ is cornered if the North Pole $\mathcal{N}$ is at the origin. In particular, $\mathcal{P}$ is contained in the closed non-positive orthant of $\mathbb{R}^{n-1}$.

A way to assemble all the constant terms of the linear equations describing an alcoved polyhedron $\mathcal{P}$ is to arrange them into a matrix $A=\left(a_{i j}\right)$ as follows:

$$
\begin{array}{r}
a_{n k} \leq x_{k} \leq-a_{k n}, \quad a_{i j} \leq x_{i}-x_{j} \leq-a_{j i}, \quad i, j, k \in[n-1], \\
a_{i i}=0, \quad i \in[n] .
\end{array}
$$

Let $M_{n}\left(\mathbb{R}_{\leq 0}\right)$ denote the set of square non-positive real matrices of order $n$. 
Definition 4.3 (Normal idempotent matrix). Consider $A=\left(a_{i j}\right) \in M_{n}\left(\mathbb{R}_{\leq 0}\right)$.

1. The matrix $A$ is called normal if $a_{i i}=0$, all $i \in[n]$, i.e., $A$ is is zerodiagonal.

2. The normal matrix $A$ is called tropically idempotent if $a_{i k}+a_{k j} \leq a_{i j} \leq 0$, all $i, j, k \in[n]$.

Remark 4.4. Let us use tropical algebra over the extended real numbers $\mathbb{R} \cup$ $\{-\infty\}$ to operate with numbers and n-dimensional vectors. The tropical operations are $\oplus:=\max$ (tropical addition) and $\odot:=+$ (tropical multiplication), coordinatewise. The element $-\infty$ is neutral for tropical addition ${ }^{1}$ For example: $(2,3) \oplus(-1,5)=(2,5),(-4) \odot(2,-\infty)=(-2,-\infty)$ and $(2,3) \odot(-1,5)^{T}=$ $\max \{1,8\}=8$. Now extend these tropical operations to matrices, following the usual way. In this context, it is well-known that any normal matrix satisfies $A \leq$ $A \odot A$. Therefore, a normal matrix $A$ is idempotent if and only if $A \odot A \leq A$, which (since tropical addition means computing a maximum) is expressed as $a_{i k}+a_{k j} \leq$ $a_{i j}$, as shown in item 2 above.

Tropical addition $\oplus=\max$ is idempotent, since $a \oplus a=a$, all $a$. Given $a \in \mathbb{R}$, no opposite element exists for a with the operation $\oplus$.

Remark 4.5. We also use classical addition of matrices, when we talk about perturbations.

Definition 4.6. $\quad$ 1. The tropical identity matrix is the square matrix $I=\left(i_{k l}\right)$ with $i_{k k}=0, i_{k l}=-\infty$, otherwise.

2. For $k, l \in[n]$, the tropical permutation matrix is the square matrix $P^{(k l)}$ obtained from I by permuting the $k-t h$ and $l-t h$ rows.

Tropically, the only invertible matrices are the diagonal ones: $D=\operatorname{diag}(b)$, with $b \in \mathbb{R}^{n}$ and $-\infty$ outside the main diagonal. Indeed, $D^{-1}=\operatorname{diag}(-b)$ is the topical inverse. The conjugate of a matrix $A$ by $D$ is

$$
{ }^{D} A:=D \odot A \odot D^{-1} .
$$

Notation 4.7. The notation ${ }^{(k l)} A$ is simplified to ${ }^{(k l)} A$.

Since any matrix can be normalized (however, not uniquely), working with normal matrices may be advantageous, and it is no serious restriction. We will do so in the sequel.

\footnotetext{
${ }^{1}$ We will use the alien element $-\infty$ as little as possible.
} 
Remark 4.8 (CORE BACKGROUND). The following are well-known facts. If $A$ is a real matrix, then the set

$$
\begin{array}{r}
\mathrm{t}-\operatorname{conv}(A):=\left\{x=\lambda_{1} \odot \operatorname{col}(A, 1) \oplus \cdots \oplus \lambda_{n} \odot \operatorname{col}(A, n): \lambda_{1}, \ldots, \lambda_{n} \in \mathbb{R}\right\} \\
=\left\{x=A \odot \lambda: \lambda \in \mathbb{R}^{n}\right\}
\end{array}
$$

of all tropical linear combinations of the columns of $A$ is a polytopal complex in $\mathbb{R}^{n}$ of impure dimension (see [20, 44]]). We have $x=\left(x_{i}\right)$ with

$$
x_{i}=\max _{k \in[n]} \lambda_{k}+a_{i k}, \quad i \in[n] .
$$

Further, if $A$ is normal idempotent then $\mathrm{t}-\mathrm{conv}(A)$ is much more than a complex: it is just one alcoved polytope (see [15, 26, 39, 48, 49]). This is a key fact. In such a case, the intersection of $\mathrm{t}-\operatorname{conv}(A)$ with the classical hyperplane $\left\{x_{n}=0\right\}$ is also an alcoved polytope and its dimension is one unit smaller. It is denoted $\mathcal{P}(A)$ in these notes:

$$
\mathcal{P}(A):=\left\{x \in \mathbb{R}^{n}: x_{n}=0\right\} \cap \mathrm{t}-\operatorname{conv}(A) \mathfrak{L}^{2}
$$

Conversely, $\mathcal{P}(A)$ determines $\mathrm{t}-\operatorname{conv}(A)$, because $\mathrm{t}-\operatorname{conv}(A)$ is closed under classical addition of the constant vector $r(1, \ldots, 1)^{T}$ (i.e., closed under tropical scalar multiplication by $r$ ), for all $r \in \mathbb{R}$.

In order to sketch the polytope $\mathcal{P}(A)$ in $\mathbb{R}^{n-1}=\left\{x_{n}=0\right\}$, we must compute the following auxiliary matrix (called geometric matrix)

$$
A_{0}=\left(\alpha_{i j}\right), \quad \alpha_{i j}:=a_{i j}-a_{n j} .
$$

In particular, the last row in $A_{0}$ is zero. Since each column of $A_{0}$ is a tropical scalar multiple of the corresponding column in $A$, we have $\mathrm{t}-\operatorname{conv}(A)=\mathrm{t}-\operatorname{conv}\left(A_{0}\right)$. The columns of $A_{0}$ belong to $\mathbb{R}^{n-1}$ and

$$
\mathcal{P}(A)=\mathcal{P}\left(A_{0}\right) .
$$

If the matrix $A$ is normal, then both $\mathrm{t}-\operatorname{conv}(A)$ and $\mathcal{P}(A)$ contain the origin ${ }^{3}$

A converse holds (see [39 48]): if $\mathcal{P} \subset \mathbb{R}^{n-1}$ is an alcoved polytope containing the origin, then there exists a normal idempotent matrix $A \in M_{n}^{N I}$ such that

$$
\mathcal{P}=\mathcal{P}(A) \text {. }
$$

Moreover, if the constant coefficients come from a normal idempotent matrix, then the inequalities are tight (see [1] 39]).

\footnotetext{
${ }^{2}$ Instead of $\mathrm{t}-\operatorname{conv}(A)$, we could use as well $\mathrm{t}-\operatorname{cone}(A)$, the definition being analogous to $\mathrm{t}-\operatorname{conv}(A)$, except that $\lambda_{j}$ runs in $\mathbb{R} \cup\{-\infty\}$. The set $\mathrm{t}-\operatorname{cone}(A)$ is a tropical cone, and the difference between both sets is just the point $(-\infty, \ldots, \infty)^{T}$. We get $\mathcal{P}(A)=\left\{x \in \mathbb{R}^{n}: x_{n}=\right.$ $0\} \cap \mathrm{t}-\operatorname{cone}(A)$.

${ }^{3}$ Indeed, for each $i \in[n]$, take $\lambda_{i}=0, \lambda_{j}<0$ and use $a_{i i}=0$ and $a_{i j} \leq 0$, to obtain $x_{i}=\max _{k \in[n]}\left\{\lambda_{k}+a_{i k}\right\}=0$.
} 
$M_{n}^{N I}$ is the set of normal idempotent matrices of order $n$. In view of 12 , we will always work in $M_{n}^{N I}$.

Definition 4.9. A normal matrix $A$ is visualized if $A=A_{0}$. Equivalently, $a_{i i}=$ $a_{n i}=0$ and $a_{i j} \leq 0$, all $i, j \in[n]$.

Remark 4.10. The union of the regions

$$
\begin{gathered}
R_{j}:=\left\{x \in \mathbb{R}^{n-1}: 0 \leq x_{j} \text { and } x_{k} \leq x_{j}, k \in[n-1]\right\}, \quad j \in[n-1], \\
R_{n}:=\left\{x \in \mathbb{R}^{n-1}: x_{k} \leq 0, k \in[n-1]\right\}
\end{gathered}
$$

is the whole $\mathbb{R}^{n-1}$.

We are dealing with zero-diagonal matrices $A$ as well as matrices with zero last row $A_{0}$, which are are related by $(10)$ and (11). The geometric matrix $A_{0}$ is used to graph the polytope of $\mathcal{P}(A) \subset \mathbb{R}^{n-1}$. The following is a characterization of a normal matrix and of a visualized matrix $A$ in terms of the location of the columns of the geometric matrix $A_{0} 4^{4}$

Lemma 4.11 (Location of columns). For a zero-diagonal matrix $A$, the following hold:

1. A is normal if and only if $\operatorname{col}\left(A_{0}, j\right) \in R_{j}$, all $j \in[n]$,

2. A is visualized if and only if $\operatorname{col}\left(A_{0}, j\right) \in R_{j} \cap R_{n}$, all $j \in[n]$.

Proof. 1. $(\Rightarrow)$ Fix $j \in[n]$. The $i$-th coordinate of $\operatorname{col}\left(A_{0}, j\right)$ is $x_{i}=\alpha_{i j}=$ $a_{i j}-a_{n j}$ and $j$-th coordinate is $x_{j}=a_{j j}-a_{n j}$. We have $x_{i} \leq x_{j}$ and $0 \leq x_{j}$ because $a_{j j}=0$ and the entries of $A$ are non-positive. Thus $\operatorname{col}\left(A_{0}, j\right) \in R_{j}$. The converse is easy.

2. $(\Rightarrow)$ By the item above, we have $\operatorname{col}\left(A_{0}, j\right) \in R_{j}$, all $j \in[n]$. The $i$-th coordinate of $\operatorname{col}\left(A_{0}, j\right)=\operatorname{col}(A, j)$ is $x_{i}=a_{i j} \leq 0$, whence $\operatorname{col}\left(A_{0}, j\right) \in$ $R_{n}$, all $j \in[n]$. The converse is easy.

The set of visualized matrices of order $n$ is a real cone of dimension $(n-$ $1)^{2}$. The subset of visualized idempotent matrices in denoted $M_{n}^{V I}$ and we have $M_{n}^{V I} \subset M_{n}^{N I}$.

Any normal idempotent matrix can be visualized, as the following lemma shows.

Lemma 4.12 (Visualizing a normal idempotent matrix). Given $A \in M_{n}^{N I}$, the matrix ${ }^{D} A$ is visualized idempotent, where $D=\operatorname{diag}(\operatorname{row}(A, n))$.

\footnotetext{
${ }^{4}$ It implies that, for a NI matrix, vertex labels in the North Cask of $\mathcal{P}$ follow the same cyclic sequence for every polyhedron, when going around $\mathcal{N}$. This will be used in p. 27
} 
Proof. If $A$ is idempotent, then so is $D \odot A \odot D^{-1}$. The diagonal of ${ }^{D} A$ is null, because the diagonal of $A$ is. The $(i, j)$-th entry is

$$
\left({ }^{D} A\right)_{i j}:=a_{n i}+a_{i j}-a_{n j}
$$

and it is non-positive, by normality of $A$. This shows normality of ${ }^{D} A$. The $(n, j)-$ th entry is $\left({ }^{D} A\right)_{n j}=a_{n n}+a_{n j}-a_{n j}=0$, since $a_{n n}=0$. This proves that ${ }^{D} A$ is visualized.

Visualization is a particular case of conjugation. In [49] this process is called visualization scaling.

Remark 4.13. The visualization of a normal matrix may not be normal. For example, $B=\left(\begin{array}{rrr}0 & 0 & 0 \\ -1 & 0 & 0 \\ -1 & -2 & 0\end{array}\right), B \odot B=\left(\begin{array}{rrr}0 & 0 & 0 \\ -1 & 0 & 0 \\ -1 & -1 & 0\end{array}\right), B_{0}=\left(\begin{array}{lll}1 & 2 & 0 \\ 0 & 2 & 0 \\ 0 & 0 & 0\end{array}\right)$. The matrix ${ }^{D} B=\left(\begin{array}{rrr}0 & 1 & -1 \\ -2 & 0 & -2 \\ 0 & 0 & 0\end{array}\right)$ is not normal.

The following statements are easy to prove. For every alcoved polytope $\mathcal{P} \subset$ $\mathbb{R}^{n-1}$, there exists a unique vector $v \in \mathbb{R}^{n-1}$ ( $v$ depending on $\mathcal{P}$ ) such that the translated polytope $t_{v}(\mathcal{P})$ is cornered. If $A \in M_{n}^{V I}$, then $\mathcal{P}(A)$ is cornered. The converse holds.

Corollary 4.14 (Unique representation). For every cornered alcoved polytope $\mathcal{P} \subset$ $\mathbb{R}^{n-1}$ there existe a unique matrix $A \in M_{n}^{V I}$ such that $\mathcal{P}=\mathcal{P}(A)$.

Definition 4.15 (More labels). Let $A \in M_{n}^{N I}$ be given and consider $\mathcal{P}=\mathcal{P}(A)$. We know that the columns of $A_{0}$ represent some vertices of $\mathcal{P}$. The vertex represented by $\operatorname{col}\left(A_{0}, j\right)$ will be denoted $j$, for $j \in[n]$. Vertices of $\mathcal{P}$ represented by columns of $A_{0}$ are called generators of $\mathcal{P}$. The columns of $\left(A^{T}\right)_{0}$ represent vertices of $\mathcal{P}$ (see [38]). The vertex of $\mathcal{P}$ represented by $\operatorname{col}\left(\left(A^{T}\right)_{0}, j\right)$ is denoted $12 \ldots j-1 j+1 \ldots n^{5}$ Vertices of $\mathcal{P}$ represented by columns of $A_{0}$ or $\left(A^{T}\right)_{0}$ are called principal. If $\mathcal{P}$ is maximal, then the number of vertices of $\mathcal{P}$ is $\left(\begin{array}{c}2 n-2 \\ n-1\end{array}\right)$ (see [20]), so that some vertices of $\mathcal{P}$ are non-principal. 6

For $n=4$, let us label the non-principal vertices. Consider $i \neq j \in[4]$. The shortest edge-path joining generators $\underline{i}$ and $\underline{j}$ contains three edges and the vertex closest to $\underline{i}$ is labeled $i \hat{j}]$ the vertex closest to $j$ is labeled $j i$ (see [26]). So, nonprincipal vertices are $\underline{12}, \underline{21}, \underline{13}, \underline{31}, \ldots, \underline{14}, \underline{41}]^{8}$

\footnotetext{
${ }^{5}$ The order of digits is unimportant.

${ }^{6}$ Recall that $\underline{123}=\mathcal{N}$ and $\underline{4}=\mathcal{S}$, i.e., the South Pole is a generator in $\mathcal{P}$.

${ }^{7}$ The order of digits is important.

${ }^{8}$ If $n>4$, we do not have a general rule to label the non-principal vertices of $\mathcal{P}$.
} 


\subsection{The 2-minors of a matrix}

In this subsection we introduce the 2-minors of the NI matrix $A$, some of which turn out to be edge-lengths of $\mathcal{P}(A)$.

Definition 4.16. 1. The difference of matrix $\left(\begin{array}{ll}a & b \\ c & d\end{array}\right)$ is $a+d-b-c .9$

2. The 2-minors of a matrix $A=\left(a_{i j}\right)$ are the differences of the order 2 submatrices of $A$. If $i, j, k, l \in[n], i \leq j$ and $k \leq l$, we write

$$
\begin{gathered}
a_{i j ; k l}:=a_{i k}+a_{j l}-a_{i l}-a_{j k}, \quad \text { and } \\
a_{i j ; k l}=-a_{j i ; k l}=-a_{i j ; l k}=a_{j i ; l k} .
\end{gathered}
$$

The 2-minors satisfy some of the usual properties of classical determinants, such as $a_{i j ; k l}=0$, if $i=j$ or $k=l$. Besides, cocycle relations hold

$$
a_{i j ; k l}=a_{i m ; k l}+a_{m j ; k l}=a_{i j ; k m}+a_{i j ; m l} .
$$

From (13), we get

$$
\left({ }^{D} A\right)_{i j}=-a_{i n ; i j}
$$

since $a_{i i}=0$, so the entries of the visualization of $A$ are certain 2-minors of $A$.

The following lemma is crucial: it is used in p. 15,23 and 25 .

Lemma 4.17 (Invariance). The 2-minors of a normal idempotent matrix are invariant under visualization.

Proof. Apply definition (14) and (15) to the entries $(13)$ and verify cancelations.

\section{From boxes to alcoved polyhedra}

In English, the regular verb to cant means to bevel, to form an oblique surface upon something. We will cant some edges in a box, making two dihedral angles of $3 \pi / 4$ radians (i.e., $135^{\circ}$ ). The result will be an alcoved polyhedron. Not every edge can be canted: only those edges not meeting the Poles can. Canting an edge depends on a parameter. The expressions canted box and perturbed box are synonyms.

Definition 5.1 (Perturbation of a VI box matrix). Given $A=\left(a_{i j}\right) \in M_{4}^{V I}$, we write

$$
B:=B\left(a_{14}, a_{24}, a_{34}\right) \text { as in (1) and (2) and } E:=B-A \text {. }
$$

We say that $\mathcal{P}(B)$ is the bounding box of $\mathcal{P}(A)$, that $B$ is the bounding box matrix of $A$. We also say that $E$ is the perturbation matrix of $A$.

\footnotetext{
${ }^{9}$ This is related to, but different from, the tropical determinant (also called tropical permanent).
} 
The box matrix $B$ is determined by the last column of the given matrix $A$. The perturbation matrix $E=\left(e_{i j}\right)$ satisfies

$$
e_{i 4}=e_{4 i}=e_{i i}=0 \text { and } e_{i j}=a_{i 4}-a_{i j} \leq 0, \text { all } i, j \in[4] .
$$

The above inequalities $e_{i j} \leq 0$ follow from the hypothesis that $A$ is visualized idempotent. The matrix $B$ is visualized idempotent but $E$ is not, in general. We have

$$
e_{i j}=-a_{i 4 ; j 4} \text { and } e_{i j ; k l}=-a_{i j ; k l} \text {, all } i, j, k, l \in[3] .
$$

Definition 5.2 (Cant and difference tuples of $E$ ). If $E$ is a $4 \times 4$ perturbation matrix as in (19), the cant tuple of $E$ is $c=\left(c_{j}\right) \in \mathbb{R}_{\leq 0}^{6}$, with $c_{1}:=e_{23}, c_{2}:=e_{13}, c_{3}:=$ $e_{12}, c_{4}:=e_{32}, c_{5}:=e_{31}$ and $c_{6}:=e_{21}$. The difference tuple is $d=\left(d_{i}\right) \in \mathbb{R}^{6}$, $d_{i}=c_{i+1}-c_{i}$, where $c_{7}=c_{1} \cdot 10$

In other words, start, say, at entry $(2,3)$-th in $E$, then go to the $(2,1)$-th entry, etc., and thus gather the entries of $E$ into the cant tuple $c$, running in a crocked closed path (see an example in p. 29). Using $e_{i 4}=e_{4 i}=0$ from (19), we get

$$
d_{I}=e_{i, i+1 ; i-1,4}, \quad I \in\{1,3,5\}, \quad d_{I}=e_{i-1,4 ; i, i+1}, \quad I \in\{2,4,6\},
$$

where $i=I \bmod 3$ and indices in $e_{i j}$ are reduced modulo 3 .

Definition 5.3 (Cant and difference tuples of $A$ ). For $A=B-E \in M_{4}^{V I}$ as in (18), the cant and difference tuples of $A$ are, by definition, the cant and difference tuples of $E{ }^{11}$

From (20) it follows

$$
d_{I}=-a_{i, i+1 ; i-1,4}, \quad I \in\{1,3,5\}, \quad d_{I}=-a_{i-1,4 ; i, i+1}, \quad I \in\{2,4,6\} .
$$

Explicitly,

$d_{1}=-a_{12 ; 34}, d_{3}=-a_{31 ; 24}, d_{5}=-a_{23 ; 14}, d_{2}=-a_{14 ; 23}, d_{4}=-a_{34 ; 12}, d_{6}=-a_{24 ; 31}$.

Example 5.4 (One cant). From a box $\mathcal{B}$, we will remove a right prism, whose base is a right isosceles triangle, legged $\left|e_{23}\right|$ (see figure 4). Given the box matrix $B=B(t)$, let us cant the top front edge of the box $\mathcal{B}=\mathcal{P}(B)$, using the parameter $e_{23}<0$ such that $\left|e_{23}\right| \leq \min \left\{\left|t_{2}\right|,\left|t_{3}\right|\right\}$. We have $A=\left(\begin{array}{rrrr}0 & t_{1} & t_{1} & t_{1} \\ t_{2} & 0 & t_{2}-e_{23} & t_{2} \\ t_{3} & t_{3} & 0 & t_{3} \\ 0 & 0 & 0 & 0\end{array}\right)$. Let $e_{i j}=0$, otherwise. The resulting polyhedron $\mathcal{P}(A)$ has one more facet than $\mathcal{B}$ and two 4-gon facets in the box $\mathcal{B}$ become 5-gon facets in $\mathcal{P}(A)$.

\footnotetext{
${ }^{10}$ In this section we take $n=4$, because we do not know how to make definition 5.2 in more generality.

${ }^{11}$ This makes sense, because a box is an unperturbed alcoved polyhedron.
} 


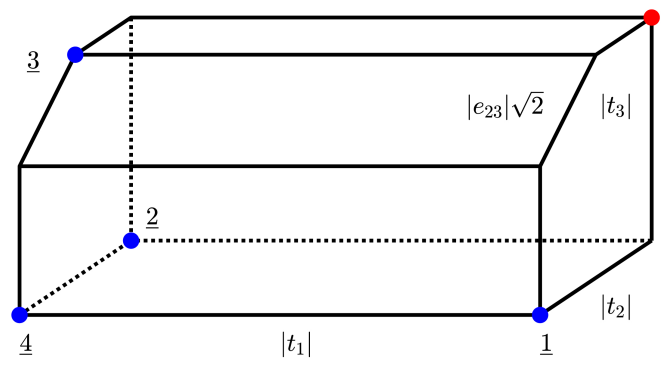

Figure 4: One cant performed on the box $\mathcal{B}=\mathcal{P}(B(t))$ yields the alcoved polyhedron $\mathcal{P}(A)$, for matrix $A$ in example 5.4 The Euclidean (resp. tropical) length of the new edge is $\left|e_{23}\right| \sqrt{2}$ (resp. $\left.\left|e_{23}\right|\right)$. The angles of the pentagonal facets in $\mathcal{P}(A)$ are $3 \pi / 4$ and $\pi / 2$. There is a left pentagon meeting $\mathcal{N}=\underline{123}$ and a right pentagon meeting $\mathcal{S}=\underline{4}$.

Definition 5.5 (Cantable edge). An edge $\ell$ in a box $\mathcal{B} \subset \mathbb{R}^{3}$ is cantable if no end point of $\ell$ is a Pole (see figure 3 right). A box has six cantable edges.

The cantable edges in a box form a Petrie polygon, i.e., a polygon dividing the box into two congruent parts. It is also a 3-equilateral spacial polygon and an antiprismatic polygon, see [43, 45]. Assuming the box matrix is (2), the equations of the cantable edges are: $\left\{x_{3}=0, x_{2}=t_{2}\right\}$ for $\ell_{1},\left\{x_{3}=0, x_{1}=t_{1}\right\}$ for $\ell_{2}$, $\left\{x_{2}=0, x_{1}=t_{1}\right\}$ for $\ell_{3}, \quad\left\{x_{2}=0, x_{3}=t_{3}\right\}$ for $\ell_{4}, \quad\left\{x_{1}=0, x_{3}=t_{3}\right\}$ for $\ell_{5}$ and $\left\{x_{1}=0, x_{2}=t_{2}\right\}$ for $\ell_{6}$.

Box, perturbation, cant and difference tuples have been defined for visualized idempotent matrices but it is good to have analogous notions for NI matrices.

Lemma 5.6 (Box and perturbation of visualization of a NI matrix). Let $V=\left(v_{i j}\right) \in$ $M_{4}^{V I}$ be the visualization of $A=\left(a_{i j}\right) \in M_{4}^{N I}$ and write $V=B-E$ (box and perturbation, as in (18)). Then

$$
v_{i j}=-a_{i 4 ; i j}, i, j \in[4] \text { and } e_{i j}=-v_{i 4 ; j 4}=-a_{i 4 ; j 4}, i, j \in[3] .
$$

In particular, $v_{i 4}=a_{i 4}+a_{4 i}, i \in[4]$, are the fourth column entries of $V$.

Proof. The first equality in (24) is (17); the second and third equalities follow from the first one, (20) and the cocycle relation (16). Alternatively, one can use lemma 4.17

The moral is that certain 2-minors of $A \in M_{4}^{N I}$ provide both the edge-lengths of the box of $\mathcal{P}(A)$ as well as the cant parameters. The following definition is a consequence of $e_{i j}=-v_{i 4 ; j 4}=-a_{i 4 ; j 4}$ in 24. 
Definition 5.7 (Perturbation, cant and difference of NI matrix). The perturbation matrix of $A \in M_{4}^{N I}$ is, by definition, the perturbation matrix $E$ of the visualization $V={ }^{D} A=B-E$ of $A$, where $D=\operatorname{diag}(\operatorname{row}(A, n))$. The cant and difference tuples of $A$ are the cant and difference tuples of $E$.

\section{Symmetries of a cube}

The contents of this section are well-known. Let $\left(u_{1}, u_{2}, u_{3}\right)$ be the canonical basis in $\mathbb{R}^{3}$. Let $\Sigma_{3}, \Sigma_{4}$ denote the permutation groups in 3,4 symbols. The unit cube centered at the origin $\mathcal{Q} \subset \mathbb{R}^{3}$ has 24 orientation-preserving symmetries (called rotations, for short). Indeed, each rotation of $\mathcal{Q}$ permutes the diagonals of $\mathcal{Q}$ (see in figure 3, center, the diagonals of a cube, labeled 1, 2, 3, 4 not underlined). The full group of symmetries of $\mathcal{Q}$ is the direct product $\mathcal{G}_{4}=\mathbb{Z}_{2} \times \Sigma_{4}$. So, in addition to rotations, $\mathcal{G}_{4}$ contains all the orientation-reversing symmetries, such as the antipodal map and orthogonal reflections on planes.

The element (id,id) $\in \mathcal{G}_{4}$ represents the identity map on the cube, while the element $\left(-\right.$ id, id) represents the antipodal map - id $: \mathcal{Q} \rightarrow \mathcal{Q}$. For $\tau \in \Sigma_{4}$, the element (id, $\tau$ ) represents the orientation-preserving symmetry permuting the diagonals of $\mathcal{Q}$ according to $\tau$. On the other hand, the element (- id, $\tau$ ) represents the orientation-reversing symmetry permuting the diagonals of $\mathcal{Q}$ according to $\tau$. In this section, we write $+\tau$ instead of $(\mathrm{id}, \tau)$, and $-\tau$ instead of $(-\mathrm{id}, \tau)$, for convenience.

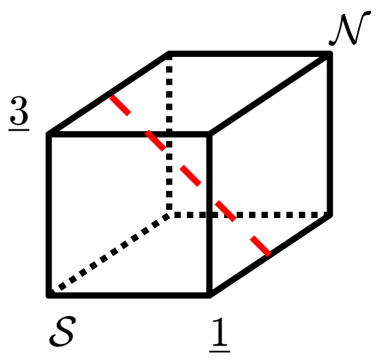

$+(13) \in \mathcal{G}_{3}$

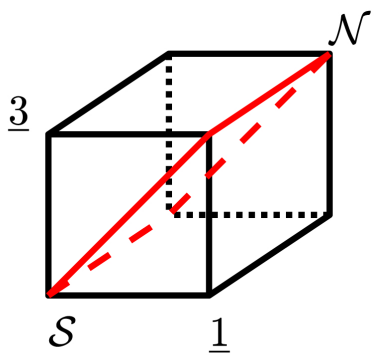

$-(13) \in \mathcal{G}_{3}$

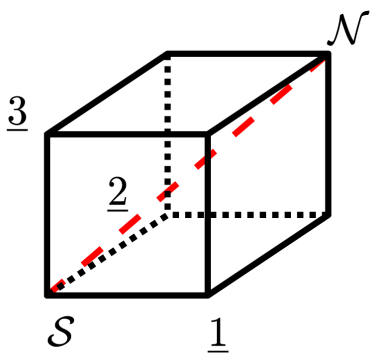

$+(123) \in \mathcal{G}_{3}$

Figure 5: A Polar Exchange rotation is $+(13)$ (left). The orthogonal reflection on plane $x_{1}=x_{3}$ is $-(13)$ (center). The $120^{\circ}$ rotation around the Polar Axis (in the positive sense) is $+(123)$ (right). The invariant sets are marked in red.

Recall that $\left(u_{1}, u_{2}, u_{3}\right)$ is the canonical basis in $\mathbb{R}^{3}$. For our purposes, we only need cube symmetries preserving the Polar Axis (which is the diagonal labeled 4) so, we work with the subgroup $\mathcal{G}_{3}:=\mathbb{Z}_{2} \times \Sigma_{3}{ }^{12}$ Since (12), (13) generate the

\footnotetext{
${ }^{12} \mathcal{G}_{3}$ is isomorphic to a dihedral group of order 12.
} 
group $\Sigma_{3}$, then $+(12),+(13),-$ id generate $\mathcal{G}_{3}$. Here is a description of these group elements, as cube symmetries:

1. for $i \neq j \in[3]$, the element $+(i j)$ permutes the diagonals $i$ and $j$ of $\mathcal{Q}$, preserving orientation. It is easily seen that it corresponds to the $180^{\circ}$ rotation around the axis line spanned by the vector $u_{i}-u_{j}$ (see red dashed line in figure 5, left). It transforms the North Pole into the South Pole. It transforms the facet $x_{i}=1$ into the facet $x_{j}=-1$ and the facet $x_{k}=1$ into the facet $x_{k}=-1$, for $i \neq k \neq j$, (see figure 5, left). Such a map is called a Polar Exchange. There are three Polar Exchanges: $+(12),+(13)$ and $+(23)$.

2. for $i \neq j \in[3]$, the element $-(i j)$ is the orthogonal reflection on the plane $x_{i}=x_{j}$. The effect on $\mathcal{Q}$ is to produce a chiral copy of $\mathcal{Q} \cdot{ }^{13}$

Remark 6.1. If the edge $\ell$ is cantable in $\mathcal{Q}$, then the edge $g(\ell)$ is cantable in $\mathcal{Q}=$ $g(\mathcal{Q})$, for all $g \in \mathcal{G}_{3} \cdot{ }^{14}$

\subsection{Matrix transformations vs. cube symmetries}

The following lemma is obvious.

Lemma 6.2. If $A \in M_{4}^{N I}$, then $A^{T} \in M_{4}^{N I}$. Furthermore, $\underline{i}_{A} \mapsto j k l{ }_{A^{T}}$ is a bijection between the sets of principal vertices of $\mathcal{P}(A)$ and $\mathcal{P}\left(A^{T}\right)$, with $\{i, j, k, l\}=$ $[4]$.

Corollary 6.3 (Antipodal map; Corollary 4 in [26]). If $A \in M_{4}^{N I}$, then $A^{T} \in M_{4}^{N I}$ and

$$
\mathcal{P}\left(A^{T}\right)=-(\mathcal{P}(A)) .
$$

In particular, the matrix $A$ is symmetric if and only if the alcoved polytope $\mathcal{P}(A)$ is central-symmetric with respect to the origin.

Example 6.4. The matrices of the centered unit cube $\mathcal{Q}$ (of edge-length 2) are $Q_{0}=\left(\begin{array}{cccc}1 & -1 & -1 & -1 \\ -1 & 1 & -1 & -1 \\ -1 & -1 & 1 & -1 \\ 0 & 0 & 0 & 0\end{array}\right), Q=\left(\begin{array}{cccc}0 & -2 & -2 & -1 \\ -2 & 0 & -2 & -1 \\ -2 & -2 & 0 & -1 \\ -1 & -1 & -1 & 0\end{array}\right) \in M_{4}^{N I}$ and the visualization of $Q$ is ${ }^{D} Q=\left(\begin{array}{cccc}0 & -2 & -2 & -2 \\ -2 & 0 & -2 & -2 \\ -2 & -2 & 0 & -2 \\ 0 & 0 & 0 & 0\end{array}\right)$.

\footnotetext{
${ }^{13}$ A chiral copy of a rubber glove $G$ is obtained by turning $G$ inside out. Alternatively, we cut $G$ along a meridian, we fold the two pieces inside out and then we glue them again. A chiral copy of the cube $\mathcal{Q}$ is obtained similarly.

${ }^{14}$ This is not true for all $g \in \mathcal{G}_{4}$; for instance, take $g=+(1234)$.
} 
The group $\mathcal{G}_{3}=\mathbb{Z}_{2} \times \Sigma_{3}$ (defined in p. 16 ) acts ${ }^{15}$ on $M_{4}^{N I}$ as follows: for $A \in M_{4}^{N I}$ and $i \neq j \in[3]$,

1. $-\mathrm{id} \cdot A:=A^{T}$,

2. $-(i j) \cdot A:={ }^{(i j)} A$, (permutation of two generators)

3. $+(i j) \cdot A:={ }^{(i j)} A^{T}$, (Polar Exchange).

Indeed, item 1 is a restatement of (25). To explain items 2 and 3 , recall that the element $+(i j) \in \mathcal{G}_{3}$ permutes the diagonals $i$ and $j$ of the cube $\mathcal{Q}$; however it does not permute the generators $\underline{i}$ and $\underline{j}$. It is the group element $-(i j)$ who does this job.

Remark 6.5 (Chirality). Let $A \in M_{4}^{N I}$. A chiral copy of $\mathcal{P}(A)$ is given by $\mathcal{P}\left({ }^{(i j)} A\right)$, for $i \neq j \in[3]$. Two chiral copies of $\mathcal{P}(A)$ are related by a power of $+(123) \in \mathcal{G}_{3}$. Here $+(123)$ is the $120^{\circ}$ rotation around the Polar Axis (in the positive sense); see figure 5 (right).

\section{Maximality}

If $\mathcal{P}$ is a maximal alcoved polyhedron in $\mathbb{R}^{3}$, then in [26] (see also [27, 53]) it is proved that

1. the $f$-vector of $\mathcal{P}$ is $\left(f_{0}, f_{1}, f_{2}\right)=(20,30,12)$; in particular $\mathcal{P}$ is a dodecahedron and it is simple (i.e., trivalent in vertices), because $20 \times 3=30 \times 2$,

2. the facets of $\mathcal{P}$ are alcoved 4-gons, 5-gons and 6-gons, with facet vector (also called $p$-vector) $p=\left(p_{4}, p_{5}, p_{6}\right)=(2,8,2),(3,6,3)$ or $(4,4,4)$, where $f_{2}=p_{4}+p_{5}+p_{6}$ and $p_{j}$ is the number of $j$-gons.

Thus, every maximal alcoved polyhedron is a dodecahedron, but the converse is not true, in general.

\section{Tropical distance}

We will use tropical distance ${ }^{16}$ to measure edge-lengths:

$$
\mathrm{d}(p, q):=\max _{i, j \in[n]}\left\{\left|p_{i}-q_{i}\right|,\left|p_{i}-q_{i}-p_{j}+q_{j}\right|\right\}, \quad p, q \in \mathbb{R}^{n} .
$$

\footnotetext{
${ }^{15}$ The action of a group $G$ on a set $S$ is a map $G \times S \rightarrow S,(g, s) \mapsto g \cdot s$, such that (a) id $\cdot s=s$, all $s \in S$ and (b) $g_{1} \cdot\left(g_{2} \cdot s\right)=\left(g_{1} g_{2}\right) \cdot s$, all $g_{1}, g_{2} \in G$, all $s \in S$.

${ }^{16} \mathrm{~d}(p, q)$ is the maximum of the Chebyshev distance $\mathrm{d}_{C h}(p, q):=\max _{i, j \in[n]}\left\{\left|p_{i}-q_{i}\right|\right\}$ and a tropical version of the Hilbert projective distance $\mathrm{d}(p, q)_{p r . H}:=\max _{i, j \in[n]}\left\{\left|p_{i}-q_{i}-p_{j}+q_{j}\right|\right\}$.
} 
For example, we have $\mathrm{d}((1,1),(0,0))=1(\operatorname{not} \sqrt{2}$ !), $\mathrm{d}((1,1,1),(0,0,0))=1$ (not $\sqrt{3} !)$, and $d((-5,-2),(-2,-5))=6$. See caption in figure 4 .

Tropical distance between integral points is just integer (i.e., lattice) distance.

This distance (and a related seminorm and norm) have been used since 1979 in [5, 16, 17, 18, 19, 20, 28, 29, 39, 40]. In the literature, they are called by various names, such as tropical Hilbert projective distance or Chebyshev distance and range seminorm. In [21] we find the Hilbert projective metric on the space of rays of a convex cone.

Remark 8.1. If $i \leq j$, then $p_{i}-q_{i}+p_{j}-q_{j}$ is the 2-minor of $\left(\begin{array}{cc}p_{i} & q_{i} \\ p_{j} & q_{j}\end{array}\right)$. In particular, if $p_{n}=q_{n}=0$, then $\mathrm{d}(p, q)$ is the maximum of the absolute values of the 2-minors of the matrix whose columns are the coordinates of $p$ and $q$.

\section{Polar Casks and Equatorial Belt}

Definition 9.1. In the alcoved polyhedron $\mathcal{P} \subset \mathbb{R}^{3}$, the North Cask (resp. South Cask) is the union of the facets of $\mathcal{P}$ containing the North Pole (resp. the South Pole).

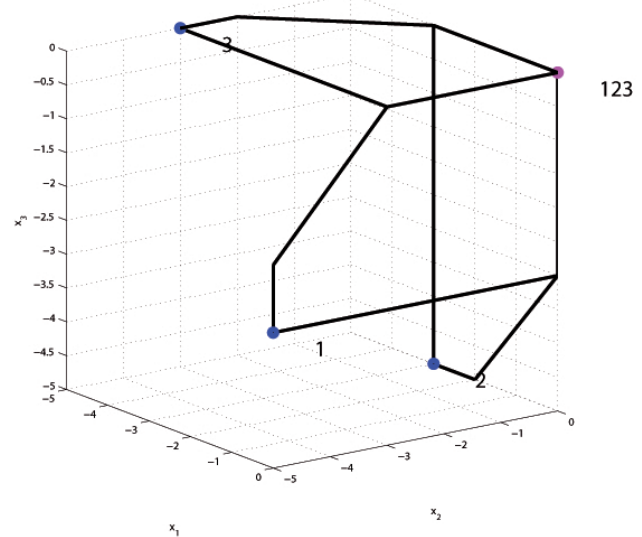

Figure 6: North Cask of type (5.5.5) left. This means that three left 5-gons meet at the North Pole. Note that the dihedral angles in the North (resp. South) Cask are $90^{\circ}$ each. Cf. figure 7 .

Definition 9.2 (Type of a Polar Cask). In a maximal alcoved dodecahedron $\mathcal{D} \subset$ $\mathbb{R}^{3}$, consider a Pole $\mathcal{V} \in\{\mathcal{N}, \mathcal{S}\}$. Let $p, q, r \in\{4,5,6\}$ with $p+q+r=15$. In $\mathcal{V}$, if the facet in $x_{1}=$ cnst is a $p-$ gon, the facet in $x_{2}=$ cnst is a q-gon and the facet in $x_{3}=$ cnst is a r-gon, and these facets meet at $\mathcal{V}$, then the type of the $\mathcal{V}$-Cask is (p.q.r). If such a pentagon is right (resp. left) then the $\mathcal{V}$-Cask is right (resp. left). 


\subsection{North Cask}

For $i \in[3]$ we consider $i-1$ and $i+1$ reduced to elements in [3] modulo 3. Notations $\rho_{i}, \lambda_{i}, \delta_{i}, \epsilon_{i}$ are introduced in the proof of the next proposition. The Greek letter $\lambda$ reminds us of left, and $\rho$ reminds us of right. These letters will represent certain non-negative edge-lengths of some facet $x_{i}=$ cnst, with $i \in[3]$ of the North Cask, where the subscripts of the letters coincide with the subscripts of the variable facet equation. Every edge-length $\rho_{i}$ (resp. $\lambda_{i}$ ) arises from canting a certain cantable edge in the bounding box. Every edge-length $\delta_{i}$ (resp. $\epsilon_{i}$ ) is the complement of a corresponding $\rho_{i}$ (resp. $\lambda_{i}$ ) in the sense that 29 ) holds.

Proposition 9.3. For a maximal alcoved dodecahedron $\mathcal{D}=\mathcal{P}(A)$ with $A \in M_{4}^{V I}$, the following hold:

1. the type of the North Cask is determined by the signs of three 2-minors of A:

$$
a_{i, 4 ; i-1, i+1}, \quad i \in[3],
$$

2. the type of the North Cask is either $(\tau(4) . \tau(5) . \tau(6))$, for all permutations $\tau$ in $\Sigma_{3}$, or (5.5.5) right, or (5.5.5) left,

3. the $f$-vector of the North Cask is $\left(f_{0}, f_{1}, f_{2}\right)=(10,12,3)$.
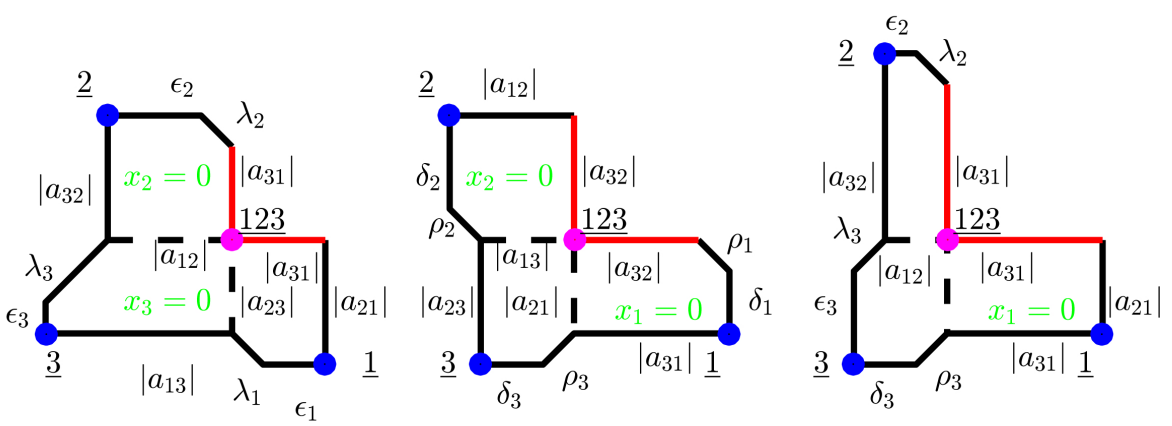

Figure 7: North Cask is (5.5.5) left (left), (5.5.5) right (center), (4.5.6) (right). Dashed segments are meant to be folded down. Red segments are meant to be glued together. If the figure on the left is folded, we get the North Cask shown in figure 6. If, instead of folding down, we fold up, we will get chiral copies. Details on the center figure are explained in remark 9.5

Proof. The matrix $A$ is visualized idempotent, whence $a_{4 i}=0, i \in[4]$, i.e., the North Pole $\mathcal{N}$ is the origin. From (14) and (15), we get

$$
a_{i, 4 ; i-1, i+1}=a_{i, i-1}-a_{i, i+1}, \quad i \in[3] .
$$


We cannot have $a_{i, 4 ; i-1, i+1}=0$, by maximality of $\mathcal{D}$ with respect to $f$-vector (i.e., the matrix $A$ is generic inside $M_{4}^{V I}$ ).

For fixed $i \in[3]$, we define

1. if $a_{i, 4 ; i-1, i+1}>0$,

(a) $\rho_{i+1}:=a_{i, 4 ; i-1, i+1}$ and $\lambda_{i-1}:=-\infty$,

(b) $\delta_{i+1}:=a_{i, i-1 ; i+1, i-1}$ and $\epsilon_{i-1}:=-\infty$,

2. if $a_{i, 4 ; i-1, i+1}<0$,

(a) $\rho_{i+1}:=-\infty$ and $\lambda_{i-1}:=-a_{i, 4 ; i-1, i+1}$,

(b) $\delta_{i+1}:=-\infty$ and $\epsilon_{i-1}:=a_{i, i+1 ; i-1, i+1}$.

Clearly,

there are exactly three positive numbers in the set $\left\{\rho_{1}, \rho_{2}, \rho_{3}, \lambda_{1}, \lambda_{2}, \lambda_{3}\right\}$.

By the determinantal properties of 2-minors and the cocycle relations (16), we have

$\delta_{i+1}+\rho_{i+1}=\left\{\begin{array}{l}-a_{i-1, i+1}, \text { if case } 1 \text { above, } \\ -\infty, \text { otherwise }\end{array} \epsilon_{i-1}+\lambda_{i-1}=\left\{\begin{array}{l}-\infty, \text { if case } 1 \text { above }, \\ -a_{i+1, i-1}, \text { otherwise },\end{array}\right.\right.$

and we say that $\delta_{i+1}, \rho_{i+1}$ (resp. $\epsilon_{i-1}, \lambda_{i-1}$ ) are complementary. Also, operating indices modulo 3 , we have

$$
\begin{gathered}
\lambda_{i+1}=\left\{\begin{array}{l}
-a_{i-1,4 ; i+1, i}=a_{i-1, i}-a_{i-1, i+1} \\
-\infty,
\end{array} \epsilon_{i+1}=\left\{\begin{array}{l}
a_{i-1, i ; i+1, i} \\
-\infty
\end{array}\right.\right. \\
\epsilon_{i+1}+\lambda_{i+1}=\left\{\begin{array}{l}
-a_{i, i+1} \\
-\infty .
\end{array}\right.
\end{gathered}
$$

Two cases arise:

- Case 1: if there exists $i \in[3]$ such that $\rho_{i+1}>0$ and $\lambda_{i+1}>0$. Then $\delta_{i+1}$ and $\epsilon_{i+1}$ are positive (this follows from $A$ being normal idempotent) and $\rho_{i+1}, \lambda_{i+1}, \delta_{i+1}, \epsilon_{i+1},\left|a_{i-1, i}\right|$ and $\left|a_{i, i-1}\right|$ are the edge-lengths of facet $x_{i+1}=0$, which makes a 6 -gon in the North Cask of $\mathcal{D}{ }^{[17}$ Geometrically, the effect on the bounding box of $\mathcal{D}$ is canting the left back edge $\ell_{3}$ by cant

\footnotetext{
${ }^{17}$ We explain the instance $i=2$, the other ones being similar (see figure 7 right). The coordinates of the generator $\underline{3}$ are $\left(\begin{array}{c}a_{13} \\ a_{23} \\ 0\end{array}\right)$ and $\delta_{3}+\rho_{3}=-a_{13}$ follows from $29, \epsilon_{3}+\lambda_{3}=-a_{23}$ follows from 31. Besides, $\rho_{3}+\left|a_{21}\right|=\left|a_{23}\right|$ follows from 27) and case 1a Similarly, we have $\lambda_{3}+\left|a_{12}\right|=\left|a_{13}\right|$.
} 
parameter $\lambda_{3}$ and the right front edge $\ell_{6}$ by cant parameter $\rho_{3}$ (recall caption in figure 3 .

Further, $\rho_{i-1}<0$ and $\lambda_{i-1}<0$ must hold, by items (1a) and (2a), in which case the facet $x_{i-1}=0$ makes a 4 -gon in the North Cask of $\mathcal{D}$, with edgelengths $\left|a_{i, i-1}\right|$ and $\left|a_{i+1, i-1}\right|$. In addition, we have $\rho_{i}>0$ or $\lambda_{i}>0$ but, because of (28), not both. Thus, the facet $x_{i}=0$ makes a 5-gon in the North Cask of $\mathcal{D}$.

- Case 2: if no $i \in[3]$ exists such that $\rho_{i+1}>0$ and $\lambda_{i+1}>0$. Then either $\rho_{i}>0$, all $i \in[3]$ or $\lambda_{i}>0$, all $i \in$ [3], because (28) holds. These two subcases are chiral to each other. A similar argument to the one given in the former case shows that the facet in $x_{i}=0$ is a 5-gon in $\mathcal{D}$. This 5-gon is left if and only if $\lambda_{i}>0$, in which case $\epsilon_{i}>0$ and $\lambda_{i}, \epsilon_{i}, \lambda_{i}+\epsilon_{i},\left|a_{i+1, i}\right|$ and $\left|a_{i+1, i-1}\right|$ are the edge-lengths. This 5-gon is right if and only if $\rho_{i}>0$ (see details in figure 7, left and center).

In either case, the North Cask of $\mathcal{D}$ consists of the three facets $x_{i}=0, i \in[3]$ as described above, all meeting at $\mathcal{N}$, the origin.

According to definition 9.2 , the possible North Cask types of $\mathcal{D}$ are

- (4.5.6) as well as all permutations of it: $(\tau(4) . \tau(5) . \tau(6)), \tau \in \Sigma_{3}$,

- (5.5.5) right, if $\rho_{i}>0$, all $i \in$ [3], or (5.5.5) left, if $\lambda_{i}>0$, all $i \in[3]$, (see figure 6 .

This proves items 1 and 2 in the proposition. Vertices, edges and facets in the North Cask of $\mathcal{D}$ are easily counted giving an $f$-vector equal to $(10,12,3)^{18}$ (see figures 6 and 7 . This proves item 3

Remark 9.4. 1. In the previous proof, we have encountered two cases:

- $\{p, q, r\}=\{4,5,6\}$,

- $6 \notin\{p, q, r\}$ and $p=q=r=5$,

the second case splitting into two mutually chiral ones.

2. Any pentagon belonging to a Polar Cask of type (5.5.5) left (resp. right) is a left (resp. right) pentagon.

3. There are exactly three positive numbers in the set $\left\{\epsilon_{1}, \epsilon_{2}, \epsilon_{3}, \delta_{1}, \delta_{2}, \delta_{3}\right\}$.

4. In $\mathcal{P}(A)$, the direction of an edge of tropical length $\rho_{i}$ or $\lambda_{i}$ (if any) is $u_{i-1}+$ $u_{i+1}$, all $i \in[3]$ (easy to check).

${ }^{18}$ Note $1=10-12+3$, i.e., a North Cask has the Euler characteristic of a closed disc. 
Remark 9.5. Here we give some details about figure 7 center. Recall that $a_{i j} \leq 0$. In the figure, we can see that

$$
\left|a_{12}\right|-\left|a_{13}\right|>0 \text { and }\left|a_{23}\right|-\left|a_{21}\right|>0 \text { and }\left|a_{31}\right|-\left|a_{32}\right|>0,
$$

or equivalently

$$
-a_{12}+a_{13}>0 \text { and }-a_{23}+a_{21}>0 \text { and }-a_{31}+a_{32}>0,
$$

which tell us that the quantities in (27) are positive, all $i \in[3]$. In other words, $\rho_{i}>$ 0 , all $i \in[3]$, whence $\lambda_{i}=-\infty$, because (28) holds. By the proof of proposition 9.3 this is the second case in Item 1 in Remark 9.4 whence the type of the North Cask is (5.5.5) right (recall $\rho$ stands for right).

Geometrically, the effect on the bounding box $\mathcal{B}$ of $\mathcal{D}$ is canting the top left edge $\ell_{2}$ of $\mathcal{B}$ by cant parameter $\rho_{2}$, canting the back bottom edge $\ell_{4}$ by cant parameter $\rho_{1}$ and canting the right front edge $\ell_{6}$ by cant parameter $\rho_{3}$ (cf. figure 3 ).

Table 2 summarizes the North Cask types of a maximal dodecahedron $\mathcal{D}$, up to permutations in $\Sigma_{3}$, for a matrix $A \in M_{4}^{V I}$.

\begin{tabular}{|lll|l|ll|}
\hline$\lambda_{2}>0$, & $\lambda_{3}>0$, & $\rho_{3}>0$ & $\mathcal{N}$ is (4.5.6) & $\lambda_{2}=-a_{34 ; 21}, \quad \lambda_{3}=-a_{14 ; 32}, \quad \rho_{3}=a_{24 ; 13}$ \\
\hline$\rho_{1}>0$, & $\rho_{2}>0$, & $\rho_{3}>0$ & $\mathcal{N}$ is (5.5.5) right & $\rho_{1}=a_{34 ; 21}, \quad \rho_{2}=a_{14 ; 32}, \quad \rho_{3}=a_{24 ; 13}$ \\
\hline$\lambda_{1}>0$, & $\lambda_{2}>0$, & $\lambda_{3}>0$ & $\mathcal{N}$ is (5.5.5) left & $\lambda_{1}=-a_{24 ; 13}, \quad \lambda_{2}=-a_{34 ; 21}, \quad \lambda_{3}=-a_{14 ; 32}$ \\
\hline
\end{tabular}

Table 2: Types of North Cask of a maximal alcoved dodecahedron. The first line shows only the identity permutation in $\Sigma_{3}$. Cf. figure 7 .

Table 3 shows the edge-lengths of the North Cask in figure 7 right, where $A \in$ $M_{4}^{V I}$ : for $i=2$, we have $\lambda_{3}=-a_{14 ; 32}=-a_{13}+a_{12}, \rho_{3}=a_{24 ; 13}=-a_{23}+a_{21}$ and for $i=1$, we have $\lambda_{2}=-a_{34 ; 21}=-a_{32}+a_{31}$ by $(30)$.

\begin{tabular}{|c|c|c|}
\hline Facet & p-gon & Edge-lenghts \\
\hline$x_{1}=0$ & 4-gon & $\left|a_{21}\right|,\left|a_{31}\right|$ \\
\hline$x_{2}=0$ & 5-gon & $\lambda_{2}, \epsilon_{2},\left|a_{32}\right|,\left|a_{12}\right|,\left|a_{31}\right|$ \\
\hline$x_{3}=0$ & 6-gon & $\left|a_{21}\right|,\left|a_{12}\right|, \lambda_{3}, \epsilon_{3}, \delta_{3}, \rho_{3}$ \\
\hline
\end{tabular}

Table 3: Edge-lengths of the North Cask of type (4.5.6) of a maximal alcoved dodecahedron $\mathcal{P}(A)$. The chirality word is left (see figures 2 and 7 right).

The previous proposition and corollary apply to visualized idempotent matrices, and the corollary below applies to NI matrices, which are more general. The corollary follows from the invariance of 2-minors proved in lemma 4.17. The moral is that the North Cask type can be read off from a NI matrix; we do not need to visualize it. 
Corollary 9.6. For a maximal alcoved dodecahedron $\mathcal{D}=\mathcal{P}(A)$ with $A \in M_{4}^{N I}$, the type of the North Cask is determined by the signs of three 2-minors of $A$ :

$$
a_{i, 4 ; i-1, i+1}, \quad i \in[3] .
$$

We can tell the type of the North Cask from the difference tuple, as the following corollary shows.

Corollary 9.7. For a maximal alcoved dodecahedron $\mathcal{D}=\mathcal{P}(A)$ with $A \in M_{4}^{V I}$, the type of the North Cask is determined by the signs of the entries of the difference tuple of $A$ with even index.

Proof. Rewrite 23) as

$d_{I+1}=a_{i, 4 ; i-1, i+1}$, with $I \in\{1,3,5\}, i=I \bmod 3$ and indices reduced modulo 3 in $a_{i j}$,

noticing that the minus sign in (23) has vanished because we have permuted two column indices. Now apply item 1 in proposition 9.3 .

Explicitly, we have

$$
d_{2}=a_{14 ; 32}, d_{4}=a_{34 ; 21}, d_{6}=a_{24 ; 13} .
$$

A simple count gives the following result.

Corollary 9.8. The number of North Cask types of maximal alcoved dodecahedra is $8=2+3$ !

The next table follows from the proof of proposition 9.3 , recalling 28 .

\begin{tabular}{|c|c|c|}
\hline $\operatorname{sign}\left(\rho_{1}, \rho_{2}, \rho_{3}, \lambda_{1}, \lambda_{2}, \lambda_{3}\right)$ & $\operatorname{sign}\left(d_{2}, d_{4}, d_{6}\right)$ & North Cask type \\
\hline$(+++--)$ & $(+++)$ & $\mathcal{N}(5.5 .5)$ right \\
\hline$(---+++)$ & $(---)$ & $\mathcal{N}(5.5 .5)$ left \\
\hline$(--+-++)$ & $(--+)$ & $\mathcal{N}(4.5 .6)$ \\
\hline$(-+-++-)$ & $(+--)$ & $\mathcal{N}(5.6 .4)$ \\
\hline$(+--+-+)$ & $(-+-)$ & $\mathcal{N}(6.4 .5)$ \\
\hline$(-++-+-)$ & $(+-+)$ & $\mathcal{N}(4.6 .5)$ \\
\hline$(+-+--+)$ & $(-++)$ & $\mathcal{N}(5.4 .6)$ \\
\hline$(++-+--)$ & $(++-)$ & $\mathcal{N}(6.5 .4)$ \\
\hline
\end{tabular}

Table 4: Types of North Cask in terms of the signs of the entries of the difference tuple with even index. 


\subsection{South Cask}

In order to study the South Cask of an alcoved polyhedron $\mathcal{P}$, all we have to do is to turn $\mathcal{P}$ around, by a Polar Exchange rotation. We have three of them $+(12),+(13),+(23) \in \mathcal{G}_{3}$ and we can choose any one.

In this section, we find the relations between the 2-minors and the difference tuples of $A$ and $S$, where the matrix $S$ is such that the North (South) Cask of $S$ is the South (North) Cask of $A$, i.e.,

$$
\sigma \in\{(12),(13),(23)\} \text { and } S={ }^{\sigma} A^{T} .
$$

The next lemmas and corollaries show what happens to matrix entries and to 2-minors under various matrix transformations. The proofs are straightforward. Recall the group action $\mathcal{G}_{3} \times M_{4}^{N I} \rightarrow M_{4}^{N I}$ described in p. 18 .

Lemma 9.9. Let $\sigma \in\{(12),(13),(23)\}$. The conjugate matrix ${ }^{\sigma} A=\left(c_{i j}\right)$ of matrix $A \in M_{4}^{N I}$ satisfies

$$
c_{i j}=a_{\sigma(i) \sigma(j)} .
$$

Corollary 9.10. Let $\sigma \in\{(12),(13),(23)\}$. If $A=\left(a_{i j}\right) \in M_{4}^{V I}$ and $V=\left(v_{i j}\right) \in$ $M_{4}^{V I}$ is the visualization of ${ }^{\sigma} A^{T}$, then

$$
v_{i j}=-a_{\sigma(i) \sigma(j) ; \sigma(i) 4}, \quad i \neq j \in[4], \quad v_{i 4}=a_{\sigma(i) 4}, \quad i \in[3] .
$$

Proof. The entries of ${ }^{\sigma} A^{T}$ are $b_{i j}=a_{\sigma(j) \sigma(i)}$ and the entries of $V$ are $v_{i j}=$ $-b_{i 4 ; i j}=-a_{\sigma(i) \sigma(j) ; \sigma(i) 4}$, by (24) and the properties of 2-minors.

Notice: the minus sign in (39) comes from visualization, $\sigma$ acts on subscripts and transposition causes exchange of row and column indices.

Corollary 9.11 (South Cask). Let $\sigma \in\{(12),(13),(23)\}$. For a maximal alcoved dodecahedron $\mathcal{D}=\mathcal{P}(A)$ and $A \in M_{4}^{N I}$, the type and edge-lengths of the South Cask are determined by the signs of three 2-minors of $A$ :

$$
a_{\sigma(i-1) \sigma(i+1) ; \sigma(i) 4}, \quad i \in[3] .
$$

Proof. By lemma 4.17, we can assume that $A$ is VI. Then, if we rotate the dodecahedron $\mathcal{D}$ by the Polar Exchange rotation $+\sigma$, it corresponds to looking at the matrix $V \in M_{4}^{V I}$ in corollary 9.10. The 2-minors we have to look at are $v_{i 4 ; i-1, i+1}=v_{i, i-1}-v_{i, i+1}=a_{\sigma(i-1) \sigma(i+1) ; \sigma(i) 4}$, the last equality by 390 and the cocycle relations (16). Then we apply proposition 9.3 .

Now we denote by $G_{3}$ the image of the following well-defined group homomorphism

$$
\Theta: \mathcal{G}_{3} \rightarrow \mathbb{Z}_{2} \times \Sigma_{6}
$$


which, on a set of generators is given by

$$
\begin{gathered}
-\mathrm{id} \mapsto+(14)(25)(36) \\
+(12) \mapsto-(26)(35) \\
+(13) \mapsto-(15)(24) \\
+(23) \mapsto-(13)(46)
\end{gathered}
$$

As a consequence, it is easily checked 19 that

$$
-(23) \mapsto-(16)(25)(34)
$$

The group $G_{3}$ is an isomorphic copy of $\mathcal{G}_{3}$ (easy to check). Two elements in $G_{3}$ are of particular relevance: $+(14)(25)(36)$, which is called the three-position shift, and $-(16)(25)(34)$ which is called the negated reverse.

We have the natural group action of $G_{3} \times \mathbb{R}^{6} \rightarrow \mathbb{R}^{6}$ : for example, $-(16)(25)(34)$. $\left(k_{1}, k_{2}, \ldots, k_{6}\right)=-\left(k_{6}, k_{5}, \ldots, k_{1}\right)$. The isomorphism $\Theta$ induces the group action $\mathcal{G}_{3} \times \mathbb{R}^{6} \rightarrow \mathbb{R}^{6}$ : for example, $-(23) \cdot\left(k_{1}, k_{2}, \ldots, k_{6}\right)=\Theta(-(23)) \cdot\left(k_{1}, k_{2}, \ldots, k_{6}\right)=$ $-\left(k_{6}, k_{5}, \ldots, k_{1}\right)$, by $(46)$.

Recall definition 5.2 .

Lemma 9.12 (Action of $\mathcal{G}_{3}$ on difference tuples). If $E$ is a $4 \times 4$ perturbation matrix and $d^{E}$ is the corresponding difference tuple, then

1. the difference tuple of matrix $E^{T}$ is the three-position shift of $d^{E}$,

2. the difference tuple of matrix ${ }^{(23)} E$ is equal to $-(13)(46) \cdot d^{E}$,

3. the difference tuple of matrix ${ }^{(23)} E^{T}$ is the reverse negated of $d^{E}$.

Proof. We prove item 3, the rest being proved similarly. The cant tuple of $E$ is $c^{E}=$ $\left(e_{23}, e_{13}, e_{12}, e_{32}, e_{31}, e_{21}\right)$ and the cant tuple of ${ }^{(23)} E^{T}$ is $c=\left(e_{23}, e_{21}, e_{31}, e_{32}, e_{12}, e_{13}\right)$. The difference tuple of $E$ is $d^{E}=\left(e_{13}-e_{23}, e_{12}-e_{13}, e_{32}-e_{12}, e_{31}-e_{32}, e_{21}-\right.$ $\left.e_{31}, e_{23}-e_{21}\right)$ and the difference tuple of ${ }^{(23)} E^{T}$ is precisely the reverse negated of $d^{E}$.

Remark 9.13 (Nice Polar Exchange). The Polar Exchange $+(23) \in \mathcal{G}_{3}$ is particularly nice in order to compute the difference tuple of the matrix $S={ }^{(23)} A^{T}$ defined in (37): by item 3 in lemma 9.12 all we have to do is to work on matrix $E$ and follow the crocked closed path given in definition in p. 14 but backwards.

Corollary 9.14. For a maximal alcoved dodecahedron $\mathcal{D}=\mathcal{P}(A)$ with $A \in M_{4}^{V I}$ the type of the South Cask is determined by the signs of the entries of the difference tuple of $A$ with odd index.

\footnotetext{
${ }^{19}$ Notice the signs in $42-\sqrt{46}$ !
} 
Proof. We choose the Polar Exchange $+(23) \in \mathcal{G}_{3}$ and consider the matrix $S=$ ${ }^{(23)} A^{T}$. From $A=B-E$, it follows that $S=B^{\prime}-{ }^{(23)} E^{T}$, where both $B$ and $B^{\prime}={ }^{(23)} B^{T}$ are box matrices. By definition 5.7 and item 3 in lemma 9.12, the difference tuples $d^{A}=d^{E}, d^{S}=d^{(23)} E^{T}$ satisfy

$$
d_{2}^{S}=-d_{5}^{A}, \quad d_{4}^{S}=-d_{3}^{A}, \quad d_{6}^{S}=-d_{1}^{A}
$$

and the result follows from corollary 9.7

From corollary 9.8 we immediately get the following.

Corollary 9.15. The number of South Cask types of a maximal alcoved dodecahedron is 8 .

The next table follows from table 4 and (47). Note that any Polar Exchange transforms a left pentagon into a right pentagon (see figure 2).

\begin{tabular}{|c|c|}
\hline$-\operatorname{sign}\left(d_{5}^{A}, d_{3}^{A}, d_{1}^{A}\right)=\operatorname{sign}\left(d_{2}^{S}, d_{4}^{S}, d_{6}^{S}\right)$ & South Cask type \\
\hline$(+++)$ & $\mathcal{S}(5.5 .5)$ right \\
\hline$(---)$ & $\mathcal{S}(5.5 .5)$ left \\
\hline$(--+)$ & $\mathcal{S}(4.5 .6)$ \\
\hline$(+--)$ & $\mathcal{S}(5.6 .4)$ \\
\hline$(-+-)$ & $\mathcal{S}(6.4 .5)$ \\
\hline$(+-+)$ & $\mathcal{S}(4.6 .5)$ \\
\hline$(-++)$ & $\mathcal{S}(5.4 .6)$ \\
\hline$(++-)$ & $\mathcal{S}(6.5 .4)$ \\
\hline
\end{tabular}

Table 5: Types of South Cask in terms of the signs of the entries of the difference tuple with odd index.

Let us finish this subsection by giving a quick method to find the type of a South Cask. Recall that we have rules to label vertices (see p. 6 and definitions 3.2 and 4.15p. In particular, non-principal vertices are labeled $i j$ and $\underline{j i}$, with $i j \neq j i$, in general. Notice that, due to the location of columns given in lemma 4.11, vertex labels in the North Cask follow the same cyclic sequence, no matter the alcoved polyhedron: going counterclockwise around $\mathcal{N}$, we visit $\underline{1}, \underline{12}, \underline{21}, \underline{2}, \underline{23}, \underline{32}, \underline{3}$, $\underline{31}$ and 13 , in this order. However, the cyclic vertex sequence in the South Cask, denoted $X$, varies from polyhedron to polyhedron. For instance $X$ may be equal to $\underline{14}, \underline{41}, \underline{124}, \underline{24}, \underline{42}, \underline{234}, \underline{34}, \underline{43}, \underline{134}$. There are $8=2^{3}$ possible sequences $X$, because $\underline{i 4}$ can be preceded or followed by $\underline{4 i}$, all $i \in[3]$, as we go around the South Pole $\mathcal{S}$ clockwise. Each such sequence can be shortened, by omitting the 3 generated vertices; for example, the former $X$ is shortened to $\underline{14}, \underline{41}, \underline{24}, \underline{42}, \underline{34}, \underline{43}$. 
For a maximal alcoved polyhedra $\mathcal{P}(A)$, look at the (shortened) sequence $X=$ $X(\mathcal{P}(A))$. For $i \in[3]$, it is easy to check that the direction of the segment $s_{i}$ joining vertices $\underline{4 i}$ and $\underline{i 4}$ in $\mathcal{P}(A)$ is either $u_{i-1}+u_{i}$ or $u_{i+1}+u_{i}$, depending on whether $\underline{i 4}$ is preceded or followed by $\underline{4 i}$ in $X$. Since the South Cask type of $\mathcal{P}(A)$ only depends on the directions of the three segments $s_{i}, i \in[3]$, then it only depends on $X$; see figure 8 .

The following table shows all the (shortened) $X$ sequences and the corresponding types of South Cask. The sequence $X$ can have zero, one, two or three inversions 20

\begin{tabular}{|c|c|c|}
\hline $14412442 \underline{34} \underline{43}$ & $\mathcal{S}(5.5 .5)$ left & 0 \\
\hline 144124424334 & $\mathcal{S}(4.6 .5)$ & 1 \\
\hline$\underline{14} \underline{41} \underline{42} \underline{24} \underline{34} \underline{43}$ & $\mathcal{S}(6.5 .4)$ & 1 \\
\hline 411424423443 & $\mathcal{S}(5.4 .6)$ & 1 \\
\hline$\underline{14} \underline{41} \underline{42} \underline{24} \underline{43} \underline{34}$ & $\mathcal{S}(5.6 .4)$ & 2 \\
\hline 411424424334 & $\mathcal{S}(4.5 .6)$ & 2 \\
\hline$\underline{41} 14 \underline{42} \underline{24} \underline{34} \underline{43}$ & $\mathcal{S}(6.4 .5)$ & 2 \\
\hline$\underline{41} 14 \underline{42} \underline{24} \underline{43} \underline{34}$ & $\mathcal{S}(5.5 .5)$ right & 3 \\
\hline
\end{tabular}

Table 6: The first column shows the (shortened) sequences $X$, the second column shows the corresponding South Cask type and the third column shows the number of inversions.
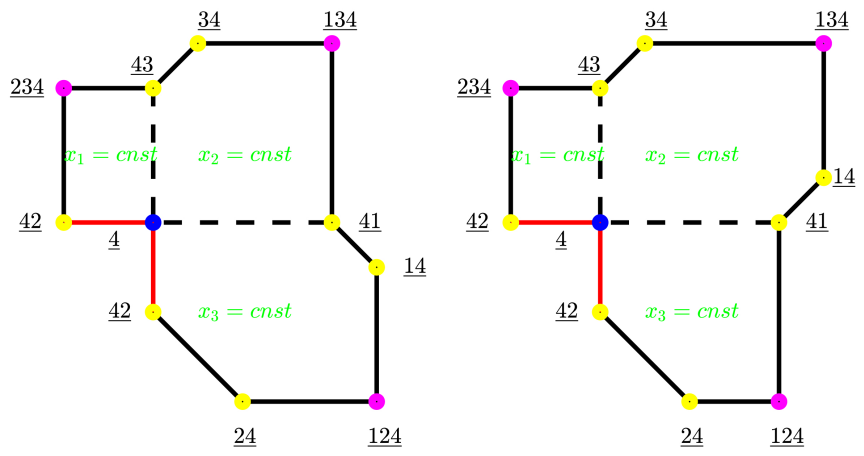

Figure 8: Two South Casks. Dashed segments are meant to be folded down. Red segments are meant to be glued together. The direction of the segment $s_{1}$ is $u_{1}+u_{2}$ (left) and $u_{1}+u_{3}$ (right). The South Cask is (4.5.6) (left) and (4.6.5) (right). The shortened sequence $X$ is $\underline{41}, \underline{14}, \underline{24}, \underline{42}, \underline{43}, \underline{34}$ (left) and $\underline{14}, \underline{41}, \underline{24}, \underline{42}, \underline{43}, \underline{34}$ (right), going clockwise.

\footnotetext{
${ }^{20} \mathrm{We}$ say that $\underline{4 i}, \underline{i 4}$ gives one inversion, and $\underline{i 4}, \underline{4 i}$ gives no inversion.
} 


\subsection{The effect of Polar Exchange on Cask types}

For $\sigma \in\{(12),(13),(23)\}$, the Polar Exchange $+\sigma \in \mathcal{G}_{3}$ transforms the facet $x_{i}=1$ into the facet $x_{j}=-1$ and the facet $x_{k}=1$ into the facet $x_{k}=-1$ in the cube $\mathcal{Q}$, when $\sigma=(i j)$. Note that any Polar Exchange transforms a left pentagon into a right pentagon. The next corollary follows.

Corollary 9.16. For $A \in M_{4}^{N I}$ and $\sigma \in\{(12),(13),(23)\}$,

1. if $\mathcal{N}$ is (5.5.5) right in $\mathcal{P}(A)$, then $\mathcal{S}$ is (5.5.5) left in the rotated polyhedron $+\sigma(\mathcal{P}(A))$,

2. if $\mathcal{N}$ is (p.q.r) in $\mathcal{P}(A)$, then $\mathcal{S}$ is $(\sigma(p) . \sigma(q) . \sigma(r))$ in the rotated polyhedron $+\sigma(\mathcal{P}(A))$.

\subsection{North and South Casks}

In this section we bring together previous results and give an example on how to compute the Cask types from the difference tuple. The following comes from corollaries 9.7, 9.14 and definition 5.7

Corollary 9.17 (Polar types determined by $\operatorname{sign}(d)$ ). For a maximal alcoved dodecahedron $\mathcal{P}(A)=\mathcal{D}$ and $A \in M_{4}^{N I}$, the types of the North and South Casks are determined by the signs of the difference tuple $d \in \mathbb{R}^{6}$. More precisely, $\operatorname{sign}\left(d_{I}\right)$, with I even, determine the type of the North Cask, and $\operatorname{sign}\left(d_{I}\right)$, with I odd, determine the type of the South Cask.

Example 9.18. Consider the VI matrix $A$ in example 2 in $p$. 34 . The box is a cube of edge-length 8 and $A=B-E$, where $E=\left(\begin{array}{rrrr}0 & -4 & -3 & 0 \\ -5 & 0 & -2 & 0 \\ -6 & -5 & 0 & 0 \\ 0 & 0 & 0 & 0\end{array}\right), c=$ $(-2,-3,-4,-5,-6,-5), d=(-1,-1,-1,-1,1,3)$ are the cant and difference tuples of $A$. To determine the North Cask type of $\mathcal{P}(A)$ we use $d_{2}<0, d_{4}<0$, $d_{6}>0$, whence the North Cask type is (4.5.6), by line 3 in table 4 To determine the South Cask type of $\mathcal{P}(A)$ we use $-\operatorname{sign}\left(d_{5}, d_{3}, d_{1}\right)=(-++)$, whence the South Cask type is (5.4.6), by line 7 in table 5 .

Corollary 9.19 (Two impossible cases). For a matrix $A \in M_{4}^{V I}$, it is impossible to have $\mathcal{N}(5.5 .5)$ right and $\mathcal{S}(5.5 .5)$ right. Similarly, it is impossible to have $\mathcal{N}$ (5.5.5) left and $\mathcal{S}$ (5.5.5) left.

Proof. We only prove the first statement. Let $c$ and $d$ be the cant and difference tuples. Now, $\mathcal{N}(5.5 .5)$ right is equivalent to $d_{I}>0, I$ even, and $\mathcal{S}(5.5 .5)$ right is equivalent to $d_{I}>0, I$ odd, (using the first lines in tables 4 and 5 ). This contradicts $\sum_{I} d_{I}=\sum_{i} c_{i}-\sum_{i} c_{i}=0$. 
The corollary above was proved in [26], Theorem 14, with a longer proof. Warning: notation disagreement: $\mathcal{S}(5.5 .5)$ left in [26] is denoted $\mathcal{S}(5.5 .5)$ right here.

\subsection{Equatorial Belt}

A box is not maximal with respect to the $f$-vector. It is the union of a North and a South Casks. In this section we present an alcoved polyhedron $\mathcal{P}$ as the union of a North and a South Casks and an Equatorial Belt. We show that this Belt is uniquely determined by the Polar Casks.

Definition 9.20 (Equatorial Belt). The Equatorial Belt of an alcoved polyhedron is the set of facets not belonging to the Polar Casks.

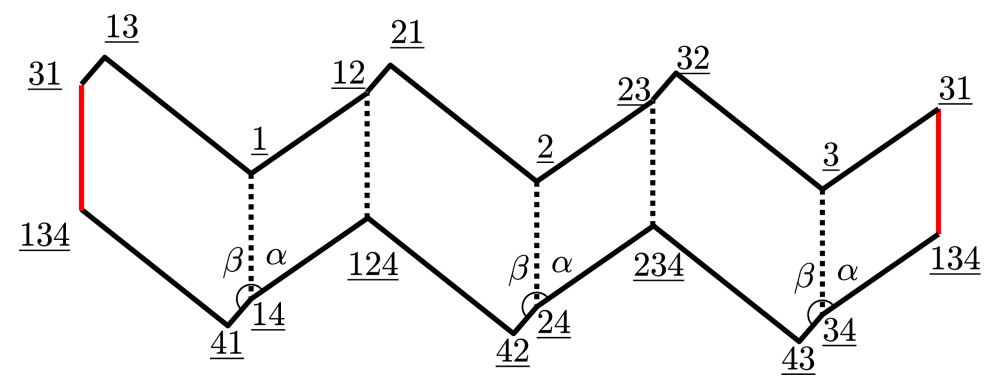

Figure 9: Equatorial belt of example 6 in p. 34. Dotted segments are meant to be folded down. Red segments are meant to be glued together. The vertical segments have direction $u_{1}+u_{2}+u_{3}$ and $\alpha=\arccos (\sqrt{1 / 3}) \simeq 54^{\circ} 44^{\prime}$, $\beta=\arccos (\sqrt{2 / 3}) \simeq 144^{\circ} 44^{\prime}$ (see angles in p. 35 .

The Equatorial Belt of a box is trivial, while the Belt of the alcoved polyhedron in example 5.4 consist of just one 4-gon facet.

Let $\left(u_{1}, u_{2}, u_{3}\right)$ be the canonical basis in $\mathbb{R}^{3}$. In a maximal alcoved dodecahedron $\mathcal{D} \subset \mathbb{R}^{3}$, the facets not belonging to the Polar Casks contain some edge in the direction $u_{1}+u_{2}+u_{3}$, and conversely. In order to get the Belt facets, we must find the edges with this direction. Clearly, three such edges are $\underline{1}-\underline{14}, \underline{2}-\underline{24}$ and $\underline{3}-\underline{34}$. These edges determine six facets in $\mathcal{D}$, which are the Belt facets of $\mathcal{D}$.

Remark 9.21. The Equatorial Belt of a maximal alcoved dodecahedron $\mathcal{D} \subset \mathbb{R}^{3}$ is uniquely determined by the Polar Casks.

Notation 9.22. Let $\mathcal{D}$ be a maximal alcoved dodecahedron. In $\mathcal{D}$, each Belt facet $F_{j}$ is the result of canting a cantable edge $\ell_{j}$ in the bounding box $\mathcal{B}, j \in[6]$ (cf. figure 3). The Belt is denoted $E B=\left(q_{1}, q_{2}, \ldots, q_{6}\right)$, where $q_{j}$ is the number of edges of $F_{j}$. By item 2 in section 7 we know that $q_{j} \in\{4,5,6\}$. 


\section{Families, group actions and quasi-Euclidean classifica- tion}

In this section, we present a classification of alcoved polyhedra that encompasses three sorts of equivalence. First, we want all boxes to be equivalent. This equivalence is, so to say, affine. Second, for two alcoved polyhedra $\mathcal{P}$ and $\mathcal{P}^{\prime}$ having the cube $\mathcal{Q}$ as bounding box, if there exists a symmetry in the group $\mathcal{G}_{3}$ taking $\mathcal{P}$ to $\mathcal{P}^{\prime}$, then we want $\mathcal{P}$ and $\mathcal{P}^{\prime}$ to be equivalent. This equivalence is, so to say, Euclidean. Third, if there is a sufficiently small perturbation taking $\mathcal{P}$ to $\mathcal{P}^{\prime}$, then we also want $\mathcal{P}$ and $\mathcal{P}^{\prime}$ to be equivalent. This equivalence is, so to say, topological.

These ideas require that we take four steps in order to define the desired equivalence relation. The second and third steps define equivalence relations, but only for polyhedra bounded by the unit cube $\mathcal{Q}$.

For a given box $\mathcal{B} \subset \mathbb{R}^{3}$, let $\mathscr{P}_{\mathcal{B}}$ be the family of all alcoved convex polytopes whose box is $\mathcal{B}$ and let $\mathscr{D}_{\mathcal{B}} \subset \mathscr{P}_{\mathcal{B}}$ be the family of all maximal alcoved dodecahedra whose box is $\mathcal{B}$. Let $\mathscr{P}$ be the family of all alcoved polyhedra and $\mathscr{D}$ the family of all maximal alcoved dodecahedra.

Definition 10.1 (First step: Equivalence of boxes). All boxes in $\mathbb{R}^{3}$ are equivalent.

Definition 10.2 (Second step: Euclidean classes in $\mathscr{P}_{\mathcal{Q}}$ and in $\mathscr{D}_{\mathcal{Q}}$ ). The group $\mathcal{G}_{3}$ acts on $\mathscr{P}_{\mathcal{Q}}\left(\right.$ resp. $\left.\mathscr{D}_{\mathcal{Q}}\right)$ and an Euclidean class in $\mathscr{P}_{\mathcal{Q}}\left(\right.$ resp. in $\left.\mathscr{D}_{\mathcal{Q}}\right)$ is a $\mathcal{G}_{3}$-orbit in $\mathscr{P}_{\mathcal{Q}}\left(\right.$ resp. $\left.\mathscr{D}_{\mathcal{Q}}\right)$.

Since the orbits of $\mathcal{G}_{3}$ have, at most 12 elements, the Euclidean classes defined above are many. Inside each of them, Euclidean angles and distances (either Euclidean or tropical) are preserved.

Definition 10.3 (Third step: Equivalence by small perturbation). Consider the matrix ${ }^{D} Q \in M_{4}^{V I}$ in example 6.4 the visualized matrix of the unit cube $\mathcal{Q}$. For $i, j \in[3], i \neq j$ and real number $\epsilon$, consider the elementary matrices $P_{i j, \epsilon}=\left(p_{k l}\right)$ with $p_{i j}=\epsilon$ and $p_{k l}=0$ whenever $(k, l) \neq(i, j)$. For $A \in M_{4}^{N I}$, define $A_{i j, \epsilon}:={ }^{D} Q+P_{i j, \epsilon}$. For $\epsilon \in[0,2]$ we have $A_{i j, \epsilon} \in M_{4}^{N I}$ (easy to prove) and for $\epsilon, \epsilon^{\prime} \in(0,2)$ we define $\mathcal{P}\left(A_{i j, \epsilon}\right)$ and $\mathcal{P}\left(A_{i j, \epsilon^{\prime}}\right)$ to be equivalent.

The extreme cases are $\epsilon=2$, providing a half-cube and $\epsilon=0$ providing the whole cube, with matrix $A_{23, \epsilon}={ }^{D} Q+P_{23, \epsilon}=\left(\begin{array}{cccc}0 & -2 & -2 & -2 \\ -2 & 0 & -2+\epsilon & -2 \\ -2 & -2 & 0 & -2 \\ 0 & 0 & 0 & 0\end{array}\right)$. The polyhedra $\mathcal{P}\left(A_{23,2}\right)$ and $\mathcal{P}\left(A_{23,0}\right)$ are not quasi-Euclidean equivalent to $\mathcal{P}\left(A_{i j, \epsilon}\right)$, for $\epsilon \in(0,2)$ (see figure 10 ).

Next, we define equivalence between polyhedra bounded by different bounding boxes, putting together the three definitions above. 

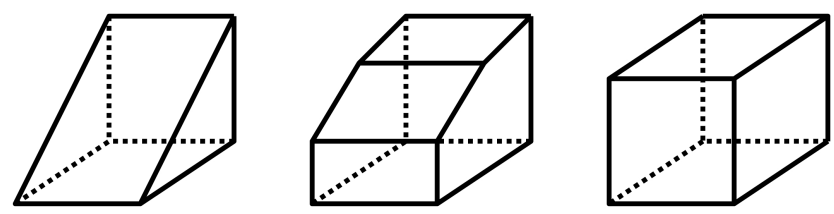

Figure 10: The three alcoved polyhedra shown are not quasi-Euclidean equivalent: they are all perturbations of the unit cube $\mathcal{Q}$.

Definition 10.4 (Fourth step: Quasi-Euclidean classes). Let $\mathcal{P}$ and $\mathcal{P}^{\prime} \subset \mathbb{R}^{3}$ be alcoved polyhedra, $\mathcal{B}$ and $\mathcal{B}^{\prime}$ be the corresponding bounding boxes. Let $f_{\mathcal{B}, \mathcal{B}^{\prime}}$ : $\mathbb{R}^{3} \rightarrow \mathbb{R}^{3}$ be the unique affine linear map taking generators to generators, i.e., $f_{\mathcal{B}, \mathcal{B}^{\prime}}(j)=\underline{j}$, for $j \in[4]$. Let $\mathcal{P}_{\mathcal{Q}}$ and $\mathcal{P}_{\mathcal{Q}}^{\prime}$ be the images under $f_{\mathcal{B}, \mathcal{Q}}$ and $f_{\mathcal{B}^{\prime}, \mathcal{Q}}$, respectively. If $\mathcal{P}_{\mathcal{Q}}$ and $\mathcal{P}_{\mathcal{Q}}^{\prime}$ are equivalent (according to definitions 10.2 and 10.3), then we define $\mathcal{P}$ and $\mathcal{P}^{\prime}$ to be quasi-Euclidean equivalent. It can be checked that this is an equivalence relation in $\mathscr{P}$ (resp. $\mathscr{D})$.

For instance, any two boxes with exactly one sufficiently small cant are quasiEuclidean equivalent.

Finally, we are ready to give the proof to theorem 1.1 .

Proof. It is enough to find exactly eight different quasi-Euclidean classes in the family $\mathscr{D}_{\mathcal{Q}}$, where $\mathcal{Q}$ is the unit cube. In order to do so, we combine North and South Casks ${ }^{21}$ getting $8^{2}=64$ possible cases. By corollary 9.19, two cases are ruled out, and we are left with 62 cases.

Consider $A \in M_{4}^{N I}$. In corollary 9.17 we have seen that the types of the North and South Casks of $\mathcal{P}(A)$ are determined by the signs of the difference tuple $d \in \mathbb{R}^{6}$ of $A$.

Consider the 62-element set $T$ consisting of all non-constant ordered tuples in $\{+,-\}^{6}$. Consider the natural group action $G_{3} \times T \rightarrow T$ (the group $G_{3}$ was defined in p.26. For example, $-(16)(25)(34) \cdot(++++--)=-(--++++)=$ $(++----)$. With a simple computer program, it is easily checked that this action has exactly 8 orbits. Each orbit corresponds to one quasi-Euclidean class and the proof of theorem 1.1 is complete.

Remark 10.5. 1. The 8 examples in subsection 10.1 belong to different classes. The cardinality of the corresponding orbits is $6,12,6,6,6,2,12$ and 12, adding up to 62.

2. The reader should not believe that the action $G_{3} \times T \rightarrow T$ used in the proof above equals the action on $T$ generated by one-shift, reverse and negate.

\footnotetext{
${ }^{21}$ By remark 9.21 the Equatorial Belt is not to be taken int account.
} 
Indeed, the subgroup of $\mathbb{Z}_{2} \times \Sigma_{6}$ generated by one-shift, reverse and negate is strictly larger than $G_{3}$.

Remark 10.6. In [26], the combinatorial classification of maximal alcoved dodecahedra is achieved attending to three vectors: $t, p$ and $h$ (which are not independent and can attain only a few integer values). Vectors $t, p, h$ can be readily obtained from the Cask types and the Equatorial Belt. Indeed, the p-vector $\left(p_{4}, p_{5}, p_{6}\right)$ (see item 2 in section 7 ) is obtained by counting 4-gons, 5-gons and 6-gons. For instance, in example 3 in $p$. 34 we have $\mathcal{N}(4.5 .6), \mathcal{S}(6.5 .4)$ and $E B=(5,4,5,5,6,5)$, whence we have a total of three 4-gons, six 5-gons and three 6-gons, so $p=(3,6,3)$.

The hexagons-vector or h-vector was defined in [26]. It is $h=\left(h_{1}, h_{2}, h_{3}, h_{4}\right) \in$ $(\mathbb{N} \cup\{0\})^{4}$, where $h_{j}$ denotes the number of maximal families consisting of $j$ pairwise adjacent hexagons. Clearly $h_{1}+2 h_{2}+3 h_{3}+4 h_{4}=p_{6}$. For instance, in example 3 in $p$. 34 we have three 6-gons not touching each other, whence $h=(3,0,0,0)$.

The type-vector or $\mathrm{t}$-vector was defined in [26]. It is $t=\left(t_{1}, t_{2}, t_{3}\right) \in(\mathbb{N} \cup$ $\{0\})^{3}$, where $t_{j}$ is the number of edges in the direction $u_{j-1}+u_{j+1}$, indices modulo 3 (recall item 4 in remark 9.4). For instance, in example 3 in $p$. 34 we have two edges in the direction $u_{1}+u_{2}\left(\right.$ resp. $\left.u_{2}+u_{3}\right)\left(\right.$ resp. $\left.u_{3}+u_{1}\right)$ whence $t=(2,2,2)$. Table 7 shows the relation between the two classifications. The combinatorial classification is coarser than the quasi-Euclidean one.

\begin{tabular}{|l||l|l|l|l|l|}
\hline QE class & QE1 & QE2 & QE3 & QE4 & QE5 \\
$p$ & $(4,4,4)$ & $(3,6,3)$ & $(3,6,3)$ & $(4,4,4)$ & $(4,4,4)$ \\
$h$ & $(0,0,0,1)$ & $(1,1,0,0)$ & $(3,0,0,0)$ & $(0,2,0,0)$ & $(0,0,0,1)$ \\
$t$ & $(0,2,4)$ & $(1,3,2)$ & $(2,2,2)$ & $(0,3,3)$ & $(11,1,4)$ \\
Comb. class & 6 & 2 & 1 & 4 & 5 \\
\hline QE class & & $Q E 6$ & $Q E 7$ & $Q E 8$ & \\
$p$ & & $(3,6,3)$ & $(2,8,2)$ & $(3,6,3)$ & \\
$h$ & & $(3,0,0,0)$ & $(0,1,0,0)$ & $(1,1,0,0)$ & \\
$t$ & & $(2,2,2)$ & $(1,2,3)$ & $(2,1,3)$ & \\
Comb. class & & 1 & 3 & 2 & \\
\hline
\end{tabular}

Table 7: This table shows the QE class, the $p, h$ and $t$ vectors and the Combinatorial class it corresponds to, according to [26].

\footnotetext{
${ }^{22}$ The $h$-vector considered here has nothing to do with the $h$-vector found in the literature on $f$-vectors.
} 


\subsection{Examples}

We give examples for all eight quasi-Euclidean Classes, indicating a VI matrix, the difference tuple, the North and South Casks, the Equatorial Belt, and the $p$-vector (defined in item 2, section 7). The bounding box is, in every case, the cube of edge-length 8 (this can be seen in the matrix last column).

$1 .\left(\begin{array}{rrrr}0 & -4 & -5 & -8 \\ -3 & 0 & -6 & -8 \\ -4 & -5 & 0 & -8 \\ 0 & 0 & 0 & 0\end{array}\right), d=(-1,-1,1,-1,-1,3), \mathcal{N}(4.5 .6), \mathcal{S}(4.5 .6)$, $E B=(4,5,6,4,5,6), p=(4,4,4)$ is an example for $Q E 1$

2. $\left(\begin{array}{rrrr}0 & -4 & -5 & -8 \\ -3 & 0 & -6 & -8 \\ -2 & -3 & 0 & -8 \\ 0 & 0 & 0 & 0\end{array}\right), d=(-1,-1,-1,-1,1,3), \mathcal{N}(4.5 .6), \mathcal{S}(5.4 .6)$, $E B=(4,5,5,5,6,5), p=(3,6,3)$ is an example for $Q E 2$

$3 .\left(\begin{array}{rrrr}0 & -5 & -6 & -8 \\ -4 & 0 & -5 & -8 \\ -3 & -4 & 0 & -8 \\ 0 & 0 & 0 & 0\end{array}\right), d=(1,-1,-1,-1,1,1), \mathcal{N}(4.5 .6), \mathcal{S}(6.5 .4)$, $E B=(5,4,5,5,6,5), p=(3,6,3)$ is an example for $Q E 3$

4. $\left(\begin{array}{rrrr}0 & -4 & -5 & -8 \\ -5 & 0 & -6 & -8 \\ -4 & -5 & 0 & -8 \\ 0 & 0 & 0 & 0\end{array}\right), d=(-1,-1,1,-1,1,1), \mathcal{N}(4.5 .6), \mathcal{S}(4.6 .5)$, $E B=(4,5,6,4,6,5), p=(4,4,4)$ is an example for $Q E 4$

5. $\left(\begin{array}{rrrr}0 & -5 & -6 & -8 \\ -6 & 0 & -5 & -8 \\ -5 & -4 & 0 & -8 \\ 0 & 0 & 0 & 0\end{array}\right), d=(1,-1,-1,1,1,-1), \mathcal{N}(4.5 .6), \mathcal{S}(5.4 .6)$, $E B=(5,4,6,4,5,6), p=(4,4,4)$ is an example for $Q E 5$

6. $\left(\begin{array}{rrrr}0 & -6 & -5 & -8 \\ -5 & 0 & -6 & -8 \\ -6 & -5 & 0 & -8 \\ 0 & 0 & 0 & 0\end{array}\right), d=(-1,1,-1,1,-1,1), \mathcal{N}(5.5 .5)$ right, $\mathcal{S}(5.5 .5)$ left, $E B=(4,6,4,6,4,6), p=(3,6,3)$ is an example for $Q E 6$ 
7. $\left(\begin{array}{rrrr}0 & -5 & -6 & -8 \\ -2 & 0 & -7 & -8 \\ -3 & -4 & 0 & -8 \\ 0 & 0 & 0 & 0\end{array}\right), d=(-1,-1,-1,-1,-1,5), \mathcal{N}(4.5 .6), \mathcal{S}(5.5 .5)$ left, $E B=(4,5,5,5,5,6), p=(2,8,2)$ is an example for $Q E 7$

8. $\left(\begin{array}{rrrr}0 & -4 & -5 & -8 \\ -3 & 0 & -6 & -8 \\ -4 & -3 & 0 & -8 \\ 0 & 0 & 0 & 0\end{array}\right), d=(-1,-1,-1,1,-1,3), \mathcal{N}(5.4 .6), \mathcal{S}(5.5 .5)$ left, $E B=(4,5,5,6,4,6), p=(3,6,3)$ is an example for $Q E 8$.

\section{Final remarks}

\subsection{Partial answers to two open questions}

Below we give answers in the case of maximal alcoved polyhedra. The case of arbitrary alcoved polyhedra follows from here (by degeneration, i.e., collapsing of vertices).

Question 1: How many distinct combinatorial types of polyhedra belong to each facet vector? (question posed in [47] p. 198). For maximal alcoved dodecahedra, the facet vectors are $p=\left(p_{4}, p_{5}, p_{6}\right)=(2,8,2),(3,6,3)$ and $(4,4,4)$ (see item 2 in section 7) and the number of combinatorial types are 1,2 and 3 resp. (so that $1+2+3=6$ ). This is readily checked in table 7] and follows from [26].

Question 2 is Dürer's Problem, also known as Shephard's conjecture (1975): Does a net exist? (question posed in [37]) The answer is yes, for every maximal alcoved dodecahedron $\mathcal{D}$. Indeed, the Equatorial Belt can be unfolded (cutting one $u_{1}+u_{2}+u_{3}$ edge) to a planar layout (cf. figure 9). Besides, planar layouts of North and South Casks exist, and the three layouts can be glued together. Then one has to check that no overlaps occur, for which one uses that facial angles of $\mathcal{D}$ are restricted, belonging to the following finite set (in degrees, rounded to minutes):

$$
\left\{90^{\circ}, 45^{\circ}, 135^{\circ}, 60^{\circ}, 120^{\circ}, 54^{\circ} 44^{\prime}, 125^{\circ} 16^{\prime}, 35^{\circ} 16^{\prime}, 144^{\circ} 44^{\prime}\right\} \text {. }
$$

The latter set arises from the set

$$
\{\arccos (0), \arccos (\sqrt{1 / 2}), \arccos (1 / 2), \arccos (\sqrt{1 / 3}), \arccos (\sqrt{2 / 3})\},
$$

which are the angles formed by pairs of vectors in $\left\{u_{i}, u_{i}+u_{j}, u_{1}+u_{2}+u_{3}\right\}$. Non overlapping is checked class by class, in the quasi-Euclidean classification.

\subsection{Alcoved polyhedra occur in nature}

We have found the combinatorial types of certain alcoved polyhedra in two instances. First, in Schlegel diagrams of soap bubbles assembled in a transparent 
container (see [23] p. 181, fig. 9.13). There, three classes of maximal dodecahedra are shown (items 3, 4 and 5 in fig. 9.13). Second, natural gas hydrates (also called clathrates) are solid compounds of small gas molecules and water. The $\mathrm{H}$ structure of clathrates (shown in figure in p. 354, bottom, in [50]) has the combinatorial type of a maximal alcoved dodecahedron.

\section{Acknowledgments}

I am deeply grateful to the referee for careful reading and interest. His/her suggestions and patience have been a great help to bring this paper to light. I also thank my friend P.L. Clavería for producing 3-D models of many alcoved dodecahedra and for checking many computations.

\section{References}

[1] S.N. Afriat, The system of inequalities $a_{r s}>X_{r}-X_{s}$, Proc. Camb. Phil. Soc. (1963), 59, 125-133.

[2] M. Akian, R. Bapat and S. Gaubert, Max-plus algebra, chapter 25 in Handbook of linear algebra, L. Hobgen (ed.) Chapman and Hall, 2007.

[3] M. Akian and S. Gaubert, Spectral theorem for convex montone homogeneous maps, and ergodic control, Nonlinear Analysis, TMA, 42, n.2, 637-679 (2003).

[4] M. Akian, S. Gaubert and C. Walsh, The max-plus Martin boundary, Documenta Math., 14, 195-240 (2009)

[5] M. Akian, S. Gaubert, V. Niţică and I. Singer, Best approximation in max-plus semimodules, Linear Algebra Appl. 435, (2011), 3261-3296.

[6] R.Bellman, Dynamic Programming, Princeton U. press, 1957

[7] B. Berthomieu and M. Menasche, An enumerative approach for analyzing time Petri nets, Proceedings IFIP, 9, Elsevier, (1983) 41-46.

[8] T.S. Blyth, Matrices over ordered algebraic structures, J. London Math. Soc. 39, (1964), 427-432.

[9] D. Bump and A. Schilling, Crystal bases: representations and combinatorics, World Scientific, 2017.

[10] E. Brugallé, Un peu de géométrie tropicale. Quadrature, 74, (2009), 10-22. 
[11] E. Brugallé, Some aspects of tropical geometry. Newsletter of the European Mathematical Society, 83, (2012), 23-28.

[12] P. Butkovič, Max-algebra: the linear algebra of combinatorics?, Linear Algebra Appl. 367, (2003), 313-335.

[13] P. Butkovič, Max-plus linear systems: theory and algorithms, (2010), Springer.

[14] P. Butkovič, Simple image set of $(\max ,+)$ linear mappings, Discrete Appl. Math. 105, (2000), 73-86.

[15] P. Butkovič, H. Schneider and S. Sergeev, Generators, extremals and bases of max-cones, Linear Algebra Appl. 421, 394-406, (2007).

[16] G. Cohen, S. Gaubert and J.P. Quadrat, Duality and separation theorems in idempotent semimodules, Lineal Algebra Appl. 379, (2004), 395-422.

[17] R. Cuninghame-Green, Minimax algebra, LNEMS, 166, Springer, 1970.

[18] R.A. Cuninghame-Green, Minimax algebra and applications, in Adv. Imag. Electr. Phys., 90, P. Hawkes, (ed.), Academic Press, 1-121, 1995.

[19] R.A. Cuninghame-Green, P. Butkovič, Bases in max-algebra, Linear Algebra Appl. 389, (2004) 107-120.

[20] M. Develin and B. Sturmfels, Tropical convexity, Doc. Math. 9, 1-27, (2004); Erratum in Doc. Math. 9 (electronic), 205-206, (2004).

[21] E. Deza and M.M. Deza, Dictionary of distances, Elsevier, 2006.

[22] C. Dima, Regular expressions with timed dominoes, in C.S. Calude et al. (Eds.), DMTCS (2003) LNCS 20371, 141-154, (2003)

[23] G. Fleck, Form, function and functioning, in [46], 171-189.

[24] I. Itenberg, E. Brugallé. B. Tessier, Géométrie tropicale. Editions de l'École Polythecnique, Paris, 2008.

[25] I. Itenberg, G. Mikhalkin and E. Shustin, Tropical algebraic geometry, Birkhäuser, 2007.

[26] A. Jiménez and M.J. de la Puente, Six combinatorial clases of maximal convex tropical polyhedra, arXiv: 1205.4162v2, 2012.

[27] M. Joswig and K. Kulas, Tropical and ordinary convexity combined, Adv. Geom. 10, (2010) 333-352. 
[28] M. Joswig, B. Sturmfels and J. Yu, Affine buildings and tropical convexity, Albanian J. Math. 1, n.4, (2007) 187-211.

[29] N. Krivulin and S. Sergeev, Tropical implementation of the Analytical Hierarchy Process decision method, arXiv:math.CO/1802.01989.

[30] T. Lam and A. Postnikov, Alcoved polytopes I, Discrete Comput. Geom. 38 n.3, 453-478 (2007).

[31] T. Lam and A. Postnikov, Alcoved polytopes II, arXiv:1202.4015v1 (2012).

[32] G.L. Litvinov, V.P. Maslov, (eds.) Idempotent mathematics and mathematical physics, Proceedings Vienna 2003, American Mathematical Society, Contemp. Math. 377, (2005).

[33] G.L. Litvinov, The Maslov dequantization, idempotent and tropical mathematics: a very brief introduction, in [32].

[34] G.L. Litvinov, S.N. Sergeev, (eds.) Tropical and idempotent mathematics, Proceedings Moscow 2007, American Mathematical Society, Contemp. Math. 495, (2009).

[35] G. Mikhalkin, Tropical geometry and its applications, in Invited lectures, v. II, Proceedings of the ICM, Madrid, 2006, (M. Sanz-Solé et al. eds.) 827-852; arXiv:math.AG/0601041, 2006.

[36] A. Miné, Domaines numériques abstraits faiblement relationnels, Thèse, L'École Polytechnique, Université Paris IX Dauphine, 2004.

[37] J. O'Rourke, Dürer's Problem, in [46], 77-86.

[38] M.J. de la Puente, Tropical linear maps on the plane, Linear Algebra Appl. 435, n. 7, (2011) 1681-1710.

[39] M.J. de la Puente, On tropical Kleene star matrices and alcoved polytopes, Kybernetika, 49, n.6, (2013) 897-910.

[40] M.J. de la Puente, Distances on the tropical line determined by two points, Kybernetika, 50, n.3, (2014) 408-335.

[41] M.J. de la Puente and P.C. Clavería, Volume of Alcoved Polyhedra and Mahler Conjecture, Proceedings ISSAC 2018, (2018), 319-326.

[42] A. Postnikov, Permutohedra, associahedra and beyond, Int. Math. Res. Notices (2009) (6): 1026-1106. DOI: https://doi.org/10.1093/imrn/rnn153

[43] V.V. Prasolov and V.M. Tikhomirov, Geometry, Translations of Mathematical Monographs 200, American Mathematical Society (July 2001). 
[44] J. Richter-Gebert, B. Sturmfels, T. Theobald, First steps in tropical geometry, in [32], 289-317.

[45] E. Schulte, Symmetry of polytopes and polyhedra, Chapter 18 in Handbook of Discrete and Computational Geometry, edited by Csaba D. Toth, Joseph O’Rourke, Jacob E. Goodman, 1997

[46] M. Senechal (ed.) Shaping Space: Exploring Polyhedra in Nature, Art, and the Geometrical Imagination, Springer, 2012.

[47] M. Senechal, The polyhedreron kingdom tomorrow, in [46], 193-200.

[48] S. Sergeev, Multiorder, Kleene stars and cyclic projectors in the geometry of max cones, in [34].

[49] S. Sergeev, H. Scheneider and P. Butkovič, On visualization, subeigenvectors and Kleene stars in max algebra, Linear Algebra Appl. 431, 2395-2406, (2009).

[50] E. Dendy Sloan Jr., Fundamental principles and applications of natural gas hydrates, Nature, 426, Nov. 2003, 353-359.

[51] D. Speyer, Tropical linear spaces, SIAM J. discrete math. 22, No. 4, (2008) $1527-1558$.

[52] D. Speyer, B. Sturmfels, Tropical mathematics, Math. Mag. 82 (2009) 163173.

[53] N.M. Tran, Enumerating polytropes, arXiv:1310.2012v4 (2016).

[54] A. Werner and J. Yu, Symmetric alcoved polytopes, The Electronic Journal of Combinatorics 21 (1) (2014), Paper 1.20, 14 pp, arXiv: 1201.4378v1 (2012).

[55] M. Yoeli, A note on a generalization of boolean matrix theory, Amer. Math. Monthly 68, n.6, (1961) 552-557.

[56] B. Yu, X. Zhao and L. Zeng, A congruence on the semiring of nomal tropical matices, Linear Alg. Appl. 555, (2018), 321-335, https:// doi .org/ $10.1016 / \mathrm{j} .1 \mathrm{aa} .2018 .06 .027$

[57] I. Zharkov, $C$ is not equivalent to $C^{-}$in its Jacobian: a tropical point of view, Int. Math. Res. Not. 3, n.6, (2015) 817-829. 